\title{
Theory of femtosecond coherent anti-Stokes Raman backscattering enhanced by quantum coherence for standoff detection of bacterial spores
}

\author{
C. H. Raymond Ooi ${ }^{1,2}$ Guy Beadie, ${ }^{3}$ George W. Kattawar, ${ }^{2}$ John F. Reintjes, ${ }^{3}$ Yuri Rostovtsev, ${ }^{1,2}$ \\ M. Suhail Zubairy, ${ }^{2}$ and Marlan O. Scully ${ }^{1,2}$ \\ ${ }^{1}$ Princeton Institute for Science of Materials and Department of Mechanical and Aerospace Engineering, Princeton University, \\ Princeton, New Jersey 08544-1009, USA \\ ${ }^{2}$ Institute for Quantum Studies and Department of Physics, Texas A\&M University, College Station, Texas 77843-4242, USA \\ ${ }^{3}$ Naval Research Laboratory, Washington, DC 20375, USA \\ (Received 7 December 2004; published 9 August 2005)
}

\begin{abstract}
Backscattered signal of coherent anti-Stokes Raman spectroscopy can be an extremely useful tool for remote identification of airborne particles, provided the signal is sufficiently large. We formulate a semiclassical theory of nonlinear scattering to estimate the number of detectable photons from a bacterial spore at a distance. For the first time, the theory incorporates enhanced quantum coherence via femtosecond pulses and a nonlinear process into the classical scattering problem. Our result shows a large backscattered signal in the far field, using typical parameters of an anthrax spore with maximally prepared vibrational coherence. Using train pulses of $1 \mathrm{kHz}$ of repetition rate each with energy of $10 \mathrm{~mJ}$, we estimate that about $10^{7}$ photons can be detected by a $1 \mathrm{~m}$ diameter detector placed $1 \mathrm{~km}$ away from the spore in the backward scattering direction. The result shows the feasibility of developing a real time remote detection of hazardous microparticles in the atmosphere, particularly biopathogenic spores.
\end{abstract}

DOI: 10.1103/PhysRevA.72.023807

PACS number(s): 42.65.Dr, 82.53.Ps, 42.25.Fx

\section{INTRODUCTION}

Coherent Anti-Stokes Raman Spectroscopy (CARS) has been widely used in molecular spectroscopy [1]. The availability of femtosecond lasers opens up new avenues for studying subpicosecond molecular dynamics [2] and time resolved CARS spectroscopy [3]. On the other hand, it has been shown that quantum coherence can dramatically increase the nonlinear response in atomic, molecular, or solid state media without significant absorption [4]. The subject of quantum coherence is the focus of broad research in quantum information [5], subfemtosecond pulse generation [6], and efficient nonlinear frequency conversion [7].

Recently, a technique combining quantum coherence and femtosecond lasers has been proposed to enhance the signal of CARS [8]. The FAST CARS (Femtosecond Adaptive Spectroscopic Techniques applied to CARS) can produce a sufficiently large signal which embodies the spectroscopic signature of a bacterial spore. The essence of the technique is the following. A pair of appropriately tailored, or adapted, femtosecond laser pulses with Rabi frequencies $\Omega_{1}$ and $\Omega_{2}$ establish a large coherence $\left|\rho_{b c}\right|$ between $|b\rangle$ and $|c\rangle$, the two vibrational ground states of a molecule (see Fig. 1). Generation of maximal coherence via fractional stimulated Raman adiabatic passage (STIRAP) has been demonstrated in rubidium vapor [9]. The two-photon resonance excites a large Raman coherence in the test molecules which is then spectrally distinguished from the background molecules excited by off-resonant fields. After some tens or hundreds of femtoseconds later (less than the coherence lifetime), a higher frequency $\nu_{3} \mathrm{UV}$ probe pulse with Rabi frequency $\Omega_{3}$ arrives and scatters from this coherence. By delaying the probe pulse, the CARS signal generated parametrically at frequency $\nu_{4} \simeq \nu_{3}+\omega_{b c}$ from the test molecules can be distin- guished from other nonresonant four-wave mixing signals of background molecules which persist for only a few femtoseconds, thus optimizing the desired signal to noise ratio. This technique would be extremely useful if one were to utilize the much weaker signal scattered in the backward direction compared to the probe pulse for standoff remote detection [10].

Different CARS configurations (BOX CARS, SCISSORS, RIKES, etc.) have been developed that use phase-matching conditions to spatially separate the CARS signal from competing sources; such as other nonlinearly generated fields, the incident laser fields, background noise, etc. Detection of the CARS signal in an off-phase-matched direction is seldom

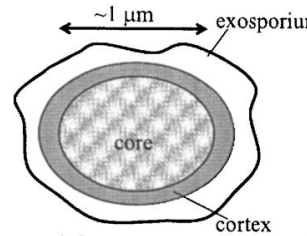

(a) spore

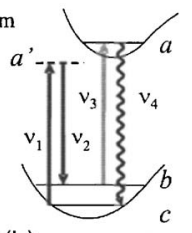

(b) CARS scheme

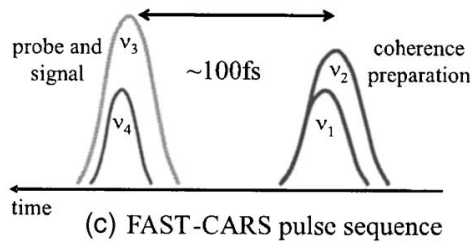

(c) FAST-CARS pulse sequence

FIG. 1. (a) The structure of a bacterial spore with the core which is approximately spherical and contains about $5 \times 10^{8}$ dipicolinic acid (DPA) molecules. (b) A simple level scheme used to describe the CARS process in a DPA molecule. (c) The time sequence of a pair of Raman pulses for coherence preparation and a probe pulse scatters off the coherence to produce a signal. 


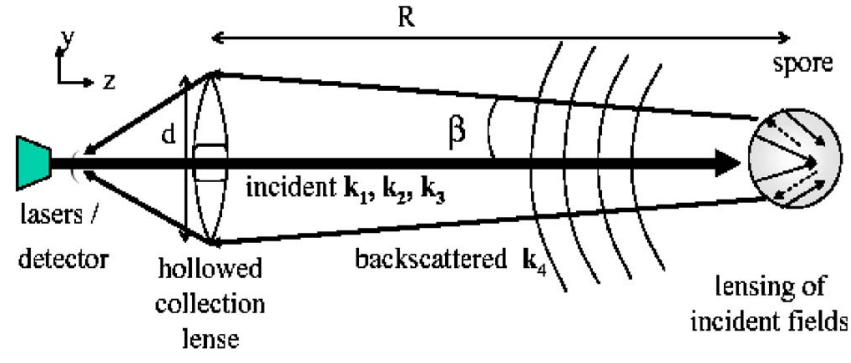

FIG. 2. (Color online) Typical setup of a femtosecond LIDAR for remote detection of backscattered CARS signal from a distant particle. The size of the spore has been exaggerated.

studied or used, because the signal is generally thought to be too weak to be measured [11]. This is true for relatively large objects. For particles with dimensions comparable to the wavelength, the phase-mismatched factor is small and does not impede detection of a useful signal. The backward CARS signal has been used in microscopy for imaging living cells [12]. This shows that non-phased-matched CARS can provide an effective, nondestructive probing method for complex molecules.

In this paper, we formulate the theory of generation of the FAST CARS signal using femtosecond laser pulses. We emphasize that we shall develop the theory for a wide class of dielectric particles composed of any molecules either in liquid or solid phase. The prime motivation is to use the backward CARS signal as a reliable spectroscopic signature for real-time remote detection of agglomerate or clump of bacterial spores in the air. As a case study, we focus on the anthrax spore Bacillus anthracis. Thus, the result of this analysis would be useful for the development of real-time preventive measures against bioterrorism using LIDAR technology. This optical technique would also be an approach for environmental studies.

In order to study the feasibility of this technique, we focus on single spore and estimate the strength of the backward signal from the spore that can be detected at a distance (Fig. $2)$. For this, we study the characteristics of the nonlinear scattering by plotting the signal (anti-Stokes) field as a function of the observation angle $\Theta$, as shown in Fig. 3. The result is encouraging. We find that the backscattered signal is almost as strong as the forward signal. The result is qualitatively similar to the experimental result of Ref. [14] who studied the angular profile using the third harmonic generation.

Detailed theory behind the result is presented in the following sections. Section II describes chemical compositions of a spore and the laser scheme used to produce the FAST CARS signal. In Sec. III, we derive the integral solution of the electric field signal outside a scattering particle with nonlinear polarization as a source. A quantum mechanical description of the nonlinear polarization is given by the antiStokes coherence $\rho_{a c}$ in Sec. IV. In Sec. V, we obtain an expression of $\rho_{a c}$ for arbitrarily short pulses. The Lorenz-Mie theory is used to describe the inhomogeneous spatial distributions of the three incident fields inside the particle as the result of refraction and internal reflection. We derive the third order susceptibility for femtosecond CARS in Sec. VI.

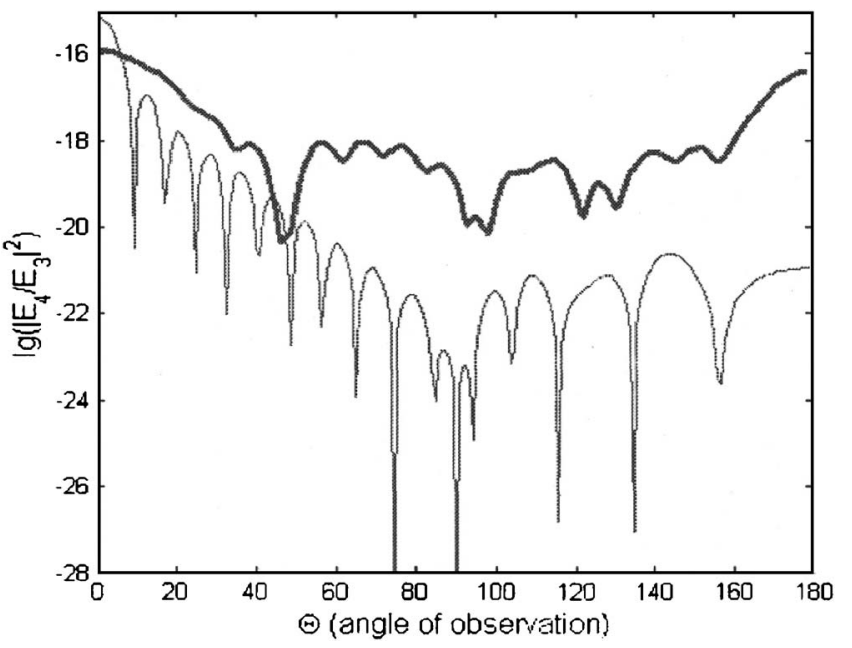

FIG. 3. Ratio of the signal intensity to probe intensity (thick line) plotted using Eq. (17) for (a) inhomogeneous incident fields (thick curve) within the spore due to lensing or focusing by the spore, including internal reflection and refraction. Refractive indices of $n_{1}=1.6, n_{2}=1.7$, and $n_{3}=1.8$ are used for the three incident fields and $n_{4}=1.6$ is used for the anti-Stokes (signal). We have used Eqs. (13) and (E6)-(E8) and take $\Phi=0$. (b) For comparison, the result for homogeneous field (thin curve) is plotted using $n_{4}=1.6$, Eqs. (22) and (23).

In Sec. VII, we give expressions for the intensity and the number of detectable photons by a typical detector at a distance. For small particle and long wavelength, analytical results of the angular dependent scattering factor for different geometries are given in Sec. VIII. Finally, Sec. IX gives the numerical results and estimates along with discussions of the physics and potential applications.

\section{MODEL FOR A SPORE AND LASER SCHEME}

The internal structure of the spore is composed of several highly developed shells of molecular structures [15]. The core of the spore is typically protected by an exosporium [Fig. 1(a)], a regularly ordered structure mainly composed of $75 \%$ amino acid tryptophan. Underneath is a three-layer coat which contains mainly proteins. The core is composed of cortex in gel phase, surrounding a cytoplasm and the nucleoid or DNA. Dipicolinic acid (DPA) and its salt calcium dipicolinate (CaDPA) are found in the cortex and cytoplasm. Raman spectroscopy has revealed characteristic peaks of $\mathrm{CaDPA}$ and proteins but can hardly distinguish the spectra of different types of matured Bacillus spores [16]. Fluorescence spectra of CaDPA has been obtained by UV irradiation and distinct UV absorption peaks of CaDPA are found around $280 \mathrm{~nm}$ and $270 \mathrm{~nm}$ [17]. The absorption maxima of the exosporium and DNA are also in the UV, around $280 \mathrm{~nm}$ and $260 \mathrm{~nm}$, respectively [15]. Further spectroscopic properties of bacterial spores can be found in Ref. [8]. All these suggest that CaDPA with the proteins can reveal the spectroscopic fingerprints of the spore when probed with UV light.

We start by modelling the spore as a sphere with homogeneously distributed DPA molecules. Although a real spore 
is typically ellipsoidal, we use spherical geometry for simplicity which enables us to incorporate the existing solutions of the classical Mie theory for a dielectric sphere to the quantum mechanical model of the three-level scheme. This is sufficient to cover all the essential physics of the problem and serves our purpose to obtain sound estimation of the backscattered signal.

The internal energy of the DPA molecule can be modelled by the three-level scheme as shown by Fig. 1(b) if we assume the lasers are close to resonances. The three-level model is a good approximation here because we are considering resonant probe laser and the coherence preparation lasers are close to resonant too. Other adjacent vibrational levels in the molecules only provide dispersive effects to the scattering processes and this is taken into account by the refractive index for each laser. This serves the purpose of estimating the number of detected photons and focusing on the essential physics. Future studies of the spectral properties would include real molecular levels.

The laser interactions are described quantum mechanically by the electric dipole interaction. The coherence between two vibrational states $b$ and $c$ in the ground electronic state of the molecule can be maximized via optimally controlled femtosecond pulses [18]. A simple quantum optical analysis with two laser pulses of Rabi frequencies $\Omega_{1}$ and $\Omega_{2}$ shows that it is possible to obtain coherence $\left|\rho_{b c}\right|$ close to the maximum value of 0.5 [19]. A probe UV laser of Rabi frequency $\Omega_{3}$ resonantly couples state $a$ to $b$, scatters off the coherence and produces a coherently enhanced CARS signal via parametric process. All three lasers are assumed to be practically collinear (Fig. 2).

Based on the standoff detection scheme, we find the ratio of the power $P_{4}$ scattered into the detector to the probe laser power $P_{3}=\frac{1}{2} \varepsilon_{0}\left|\mathbf{E}_{3}\right|^{2} c A$ as

$$
\frac{P_{4}(t)}{P_{3}}=2 \pi \frac{d^{2} \lambda^{2}}{8 R^{2} A}\left(\frac{3}{8 \pi} \frac{\gamma_{r}}{\gamma_{a c}} N\left|\rho_{b c}(t)\right| F\right)^{2},
$$

where $\left|\rho_{b c}(t)\right|$ is the magnitude of coherence between level $b$ and $c$ established via the FAST CARS [8], $\gamma_{r}$ is the radiative decay rate of level $a, \gamma_{a c}$ is the decoherence rate, $d$ is the diameter of detector, $R$ is the distance between the spore and detector and $A=\pi w^{2} / 4$ is the beam cross section. The dimensionless factor $F$ is highly dependent on the detection angle relative to the probe laser, refractive index of the spore, wavevector mismatch, spore geometry and orientation (for nonspherical spore).

\section{INTEGRAL SOLUTION OF THE SCATTERED FIELD IN FAR ZONE}

The physical process of the nonlinear scattering by a particle can be described as follows. Nonlinear interaction of the laser fields and particles creates electric polarization $\mathbf{P}$ which acts as a source to generate the CARS (anti-Stokes) signal $\mathbf{E}_{4}$. The dynamics is governed by the inhomogeneous vector wave equation valid for a nonmagnetic medium with no free charge and no free current

$$
\left(\nabla^{2}-\frac{1}{c^{2}} \frac{\partial^{2}}{\partial t^{2}}\right) \mathbf{E}_{4}(\mathbf{R}, t)=-\frac{1}{\varepsilon_{0}}\left(\boldsymbol{\nabla} \boldsymbol{\nabla} \cdot-\frac{1}{c^{2}} \frac{\partial^{2}}{\partial t^{2}}\right) \mathbf{P}(\mathbf{R}, t) .
$$

The formal solution of Eq. (2) is

$$
\mathbf{E}_{4}(\mathbf{R}, t)=\left(\boldsymbol{\nabla} \boldsymbol{\nabla} \cdot-\frac{1}{c^{2}} \frac{\partial^{2}}{\partial t^{2}}\right) \int_{V} \frac{\mathbf{P}\left(\mathbf{r}^{\prime}, t-\frac{\left|\mathbf{R}-\mathbf{r}^{\prime}\right|}{c}\right)}{4 \pi \varepsilon_{0}\left|\mathbf{R}-\mathbf{r}^{\prime}\right|} d^{3} r^{\prime},
$$

where the $\boldsymbol{\nabla}$ acts on the observation coordinates $\mathbf{R}$, and $V$ is the integration volume which includes $\mathbf{R}$ and the source (spore). Equation (3) gives the electric field at any time $t$ and space $\mathbf{R}$ outside the source due to the polarization at the whole region of the source $\mathbf{r}^{\prime}$ at some retarded time $t-\mid \mathbf{R}$ $-\mathbf{r}^{\prime} \mid / c$.

Note Eq. (3) implicitly includes reflection and refraction due to the linear response of the signal since the dielectric function $\varepsilon(\mathbf{r}, \omega)=1+\chi^{(1)}(\mathbf{r}, \omega)$ and $\mathbf{E}_{4}$ are contained in $\bar{\rho}_{\mathrm{ac}}$ which can be obtained from the solutions of the Bloch equations. In order to separate out $\mathbf{E}_{4}$ from $\bar{\rho}_{a c}$ we need to reformulate the vector wave equation. In frequency space, we use $\widetilde{\mathbf{P}}(\mathbf{R}, \omega)=\varepsilon_{0} \chi^{(1)}(\omega) \widetilde{\mathbf{E}}(\mathbf{R}, \omega)+\widetilde{\mathbf{P}}^{N L}(\mathbf{R}, \omega)$ and $\boldsymbol{\nabla} \cdot \widetilde{\mathbf{E}}=-\boldsymbol{\nabla} \cdot \widetilde{\mathbf{P}}^{N L} /$ $\varepsilon_{0} \varepsilon(\omega)$ to rewrite Eq. (2) as

$$
\left(\nabla^{2}+\frac{\omega^{2}}{c^{2}} \varepsilon(\omega)\right) \widetilde{\mathbf{E}}_{4}(\mathbf{R}, \omega)=-\frac{1}{\varepsilon_{0}}\left(\frac{\nabla \nabla \cdot}{\varepsilon(\omega)}+\frac{\omega^{2}}{c^{2}}\right) \mathbf{P}^{N L}(\mathbf{R}, \omega)
$$

which has the solution for the anti-Stokes signal [12]

$$
\widetilde{\mathbf{E}}_{4}(\mathbf{R}, \omega)=\left(\frac{\boldsymbol{\nabla} \boldsymbol{\nabla} \cdot}{\varepsilon(\omega)}+\frac{\omega^{2}}{c^{2}}\right) \int_{V} \frac{\widetilde{\mathbf{P}}^{N L}\left(\mathbf{r}^{\prime}, \omega\right) e^{i k_{4}(\omega)\left|\mathbf{R}-\mathbf{r}^{\prime}\right|}}{4 \pi \varepsilon_{0}\left|\mathbf{R}-\mathbf{r}^{\prime}\right|} d^{3} r^{\prime},
$$

where $k_{4}(\omega)=(\omega / c) \sqrt{\varepsilon(\omega)}$ and $\varepsilon(\omega)$ is the dielectric function.

Equation (5) is exact symbolically but not readily useful for computation of the signal. In the far field, we may use $\left|\mathbf{R}-\mathbf{r}^{\prime}\right| \simeq R-\hat{\mathbf{R}} \cdot \mathbf{r}^{\prime}, \quad \boldsymbol{\nabla} \boldsymbol{\nabla} \cdot\left[\tilde{\mathbf{P}}^{N L}\left(\mathbf{r}^{\prime}, \omega\right) e^{i k_{4}(\omega)\left|\mathbf{R}-\mathbf{r}^{\prime}\right|} /\left|\mathbf{R}-\mathbf{r}^{\prime}\right|\right]$ $\simeq-(\hat{\mathrm{R}} / R) k_{4}(\omega)^{2} \widetilde{P}_{R}^{N L} e^{i k_{4}(\omega)\left(R-\hat{\mathrm{R}} \cdot \mathbf{r}^{\prime}\right)} \quad$ and $\quad \widetilde{\mathbf{P}}^{N L}\left(\mathbf{r}^{\prime}, \omega\right)=\hat{\mathrm{R}} \widetilde{P}_{R}^{N L}$ $+\hat{\Theta} \widetilde{P}_{\Theta}^{N L}+\hat{\Phi} \widetilde{P}_{\Phi}^{N L}$ to rewrite Eq. (3) as

$$
\widetilde{\mathbf{E}}_{4}(\mathbf{R}, \omega)=\frac{\omega^{2}}{c^{2}} \int_{V} \frac{\left(\hat{\Theta} \widetilde{P}_{\Theta}^{N L}+\hat{\Phi} \widetilde{P}_{\Phi}^{N L}\right) e^{i k_{4}(\omega)\left(R-\hat{\mathbf{R}} \cdot \mathbf{r}^{\prime}\right)}}{4 \pi \varepsilon_{0}\left|\mathbf{R}-\mathbf{r}^{\prime}\right|} d^{3} r^{\prime},
$$

where $\widetilde{P}_{\Theta}^{N L}=\hat{\Theta} \cdot \mathbf{P}^{N L}$ and $\widetilde{P}_{\Phi}^{N L}=\hat{\Phi} \cdot \mathbf{P}^{N L}$. Note that only the transverse (angular) components of the polarization contribute to the far field. These components are related to the Cartesian components $\widetilde{P}_{q}^{N L}$ by the transformation unit vectors $\hat{\Theta}=(\cos \Theta \cos \Phi, \cos \Theta \sin \Phi,-\sin \Theta)$ and $\hat{\Phi}=(-\sin \Phi$, $\cos \Phi, 0)$. Hence, we can express Eq. (6) in the final form

$$
\begin{aligned}
\widetilde{\mathbf{E}}_{4}(\mathbf{R}, \omega)= & \frac{e^{i k_{4}(\omega) R}}{4 \pi \varepsilon_{0} R} \frac{\omega^{2}}{c^{2}} \sum_{l, p=x, y, z} \hat{l} W_{l p} \int_{0}^{\rho_{0}} d \rho \rho^{2} \int_{0}^{\pi} d \theta \sin \theta \\
& \times \int_{0}^{2 \pi} d \phi \widetilde{P}_{p}^{N L}\left(\mathbf{r}^{\prime}, \omega\right) \\
& \times e^{-i k_{4}(\omega) \rho[\cos \Theta \cos \theta+\cos (\phi-\Phi) \sin \Theta \sin \theta],}
\end{aligned}
$$




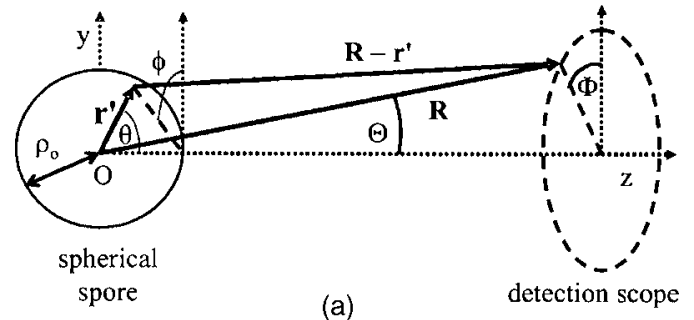

(a)

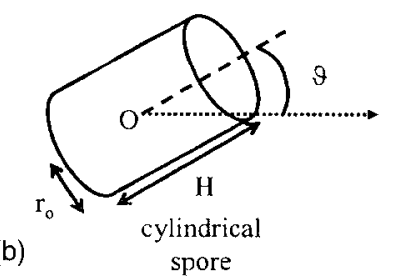

FIG. 4. (a) Schematic in spherical coordinate system used to calculate $\widetilde{\mathbf{E}}_{4}$ the signal field at the observation point $(R, \Theta, \Phi)$ produced from a spore modelled as a sphere. (b) Dimensions and orientation of a cylindrical spore.

where the angular matrix element in Cartesian coordinate is $W_{l p}=(\hat{\Theta} \cdot \hat{1})(\hat{\Theta} \cdot \hat{\mathrm{p}})+(\hat{\Phi} \cdot \hat{\mathbf{l}})(\hat{\Phi} \cdot \hat{\mathrm{p}})$ and we have used $\mathbf{R}$ $=R(\sin \Theta \cos \Phi, \sin \Theta \sin \Phi, \cos \Theta)$ and $\mathbf{r}^{\prime}=\rho(\sin \theta \cos \phi$, $\sin \theta \sin \phi, \cos \theta$ ) (see Fig. 4).

\section{QUANTUM MECHANICAL DESCRIPTION OF NONLINEAR POLARIZATION}

The incident laser fields undergo nonlinear interactions with the molecules in the particle, giving rise to the third order polarization that is related to $\widetilde{\rho}_{\mathrm{ac}}^{(3)}$ the nonlinear part of the density matrix element corresponding to the anti-Stokes emission,

$$
\widetilde{P}_{p}^{N L}\left(\mathbf{r}^{\prime}, \omega\right)=\sum_{j} \wp_{p, c a}^{(j)} \widetilde{\rho}_{a c}^{(3)}\left(\mathbf{r}_{j}^{\prime}, \omega\right) \delta\left(\mathbf{r}^{\prime}-\mathbf{r}_{j}\right),
$$

where $\wp_{p, c a}^{(j)}=\left(\mathbf{d}^{(j)} \cdot \hat{e}_{p}\right)_{c a}$. For a large number of molecules with a homogeneous number density $\eta$ in a volume $V$, we can convert the summation to integration via $\Sigma_{j} \rightarrow \eta \int_{V^{\prime}} d^{3} r_{j}$. Assuming that each molecule has the same dipole moment, we have

$$
\widetilde{P}_{p}^{N L}\left(\mathbf{r}^{\prime}, \omega\right)=\eta \wp_{p, c a} \widetilde{\rho}_{a c}^{(3)}\left(\mathbf{r}^{\prime}, \omega\right) .
$$

Using Eq. (9), the signal field can be written in a more suggestive form

$$
\widetilde{\mathbf{E}}_{4}(\mathbf{R}, \omega)=\hat{\varepsilon}_{4}(\Theta, \Phi) e^{i k_{4}(\omega) R} \frac{\mu_{0} \wp_{c a} \omega^{2}}{4 \pi R} N S(\Theta, \Phi, \omega)
$$

with

$$
\begin{aligned}
S(\Theta, \Phi, \omega)= & \frac{1}{V} \int_{0}^{\rho_{0}} d \rho \rho^{2} \int_{0}^{\pi} d \theta \sin \theta \int_{0}^{2 \pi} d \phi \\
& \times \widetilde{\rho}_{a c}^{(3)}\left(\mathbf{r}^{\prime}, \omega\right) e^{-i k_{4}(\omega) \rho[\cos \Theta \cos \theta+\cos (\phi-\Phi) \sin \Theta \sin \theta]},
\end{aligned}
$$

where

$$
\hat{\varepsilon}_{4}(\Theta, \Phi)=\sum_{l, p=x, y, z} \hat{l} W_{l, p} \frac{\wp_{p, c a}}{\wp_{c a}}
$$

gives the polarization of the signal field and depends on the detection direction specified by $\Theta, \Phi$ in spherical coordinate (see Fig. 4), $N=\eta V$ is the number of molecules in the volume $V$ of the spore, $\wp_{c a}$ and $\gamma_{a c}$ are the mean dipole moment and decoherence rate of the anti-Stokes transition, respectively.

\section{INCLUSIONS OF FEMTOSECOND PULSES AND LORENZ-MIE THEORY}

A challenging task of FAST CARS is to engineer optimum set of short probe pulses to maximize the energy generation of the anti-Stokes signal. Two decoherence lifetimes must be taken into account, the Raman $\gamma_{b c}^{-1} \sim 10^{-12} \mathrm{~s}$ and the anti-Stokes $\gamma_{a c}^{-1} \sim 10^{-13} \mathrm{~s}$. There are several competing processes that may screen out the CARS signal. For example, (i) resonant two-photon absorption, (ii) off-resonant CARS, and (iii) off-resonant $\delta_{R}$ vibrational (Raman) excitations due to background species. The first can be suppressed by having a detuning $\Delta$ from an excited electronic state. This can be achieved with optical fields due to the high lying first excited electronic state of DPA. It is well known [20] that the offresonant signals decay faster than the resonant signal which is characterized to a large extent by intramolecular vibrational redistribution (IVR). Thus, the ratio of the resonant to nonresonant signal can be optimized by choosing an appropriate delay $t_{d}$ between the probe and the Raman pulses $\delta_{R}^{-1}<t_{d}<\gamma_{b c}^{-1}$.

The expression of the Raman coherence $\rho_{b c}$ for short pulses is derived in Appendix $\mathrm{C}$ and the anti-Stokes coherence $\rho_{a c}^{(3)}$ is derived in Appendix D using the density matrix equations for the three level system given in Appendix B. At temperature around $300 \mathrm{~K}$ almost all molecules are in the ground vibrational state $|c\rangle$. We then have $\rho_{c c}(0)=1$ and $\bar{\rho}_{b c}(0)=\bar{\rho}_{a b}(0)=\bar{\rho}_{a c}(0)=0$. However, as the Raman pulses are arrive, some population is transferred from $|c\rangle$ to $|b\rangle$, with essentially no population in $|a\rangle$. The Raman coherence $\left|\rho_{b c}\right|$ would end up to be a maximum $(1 / 2)$ if the populations in both $|b\rangle$ and $|c\rangle$ becomes equal $\left(w_{b b} \simeq w_{c c} \simeq-1 / 2\right)$.

From Eq. (D3), we may rewrite Eq. (11) as

$$
\begin{aligned}
S(\Theta, \Phi, \omega)= & i \sum_{q, r, s} F_{q r s}(\Theta, \Phi, \omega) \frac{\wp_{s, a b}}{\hbar\left(\gamma_{a c}-i \Delta_{a c}\right)} \\
& \times\left.\sum_{n=0} \frac{1}{n !} \frac{d^{n} E_{3 s}(t)}{d t^{n}}\right|_{t_{3} m=0} ^{n} a_{m n}\left(t_{3}\right) i^{n-m} \\
& \times \frac{d^{n-m} \bar{\rho}_{b c, q r}\left(\Delta_{a c}\right)}{d \Delta_{a c}^{n-m}}
\end{aligned}
$$

with the dimensionless geometrical and orientation factor 


$$
\begin{aligned}
F_{q r s}(\Theta, \Phi, \omega)= & \frac{1}{V} \int_{0}^{\rho_{0}} d \rho \rho^{2} \int_{0}^{\pi} d \theta \sin \theta \int_{0}^{2 \pi} d \phi \\
& \times u_{1, q}\left(\mathbf{r}^{\prime}\right) u_{2, r}^{*}\left(\mathbf{r}^{\prime}\right) u_{3, s}\left(\mathbf{r}^{\prime}\right) \\
& \times e^{-i k_{4}(\omega) \rho[\cos \Theta \cos \theta+\cos (\phi-\Phi) \sin \Theta \sin \theta]} .
\end{aligned}
$$

Before Eqs. (10), (12), and (13) can be readily used to compute the angular distribution of the intensity, we need to obtain expressions for $u_{1, q}, u_{2, r}$, and $u_{3, s}$.

The dense protein compositions of the spore creates linear dispersions to the three incident laser fields $\widetilde{E}_{f, q}$ where $f \in 1,2,3$. As the result, the fields in the spore undergo multiple internal reflections and interference, thus acquiring a strong spatial dependence (lensing or focusing effect). This is taken into account by using the Lorenz-Mie theory (Appendix E). We can justify the use of field independent dielectric functions $\varepsilon_{i}(\omega)$ in the Lorenz-Mie theory by considering the field intensity regime where the Kerr effect is negligible. In order to incorporate the Lorenz-Mie theory, the incident fields can be decomposed into the temporal and/or spectral part and the spatial part by writing $\Omega_{f}(\mathbf{r}, t)$ $=\Sigma_{s} u_{f, s}(\mathbf{r}) E_{f s}(t) \wp_{s, a b(a c)} / \hbar$, where the spatial part is a normalized Cartesian component $u_{f, q}(\mathbf{r})=\widetilde{E}_{f, q} / \widetilde{E}_{f 0}(\omega)$ with $\widetilde{E}_{f, q}$ related to the spherical components $\widetilde{E}_{f, r}, \widetilde{E}_{f, \theta}, \widetilde{E}_{f, \phi}$ given by Eqs. (E6)-(E8) in Appendix E. The explicit relations to Cartesian components are given by $\widetilde{E}_{1, x}\left(\mathbf{r}^{\prime}, \omega\right)=\widetilde{E}_{1, r} \sin \theta \cos \phi$ $+\widetilde{E}_{1, \theta} \cos \theta \cos \phi-\widetilde{E}_{1, \phi} \sin \phi, \quad \widetilde{E}_{1, y}\left(\mathbf{r}^{\prime}, \omega\right)=\widetilde{E}_{1, r} \sin \theta \sin \phi$ $+\widetilde{E}_{1, \theta} \cos \theta \sin \phi+\widetilde{E}_{1, \phi} \cos \phi, \quad$ and $\quad \widetilde{E}_{1, z}\left(\mathbf{r}^{\prime}, \omega\right)=\widetilde{E}_{1, r} \cos \theta$ $-\widetilde{E}_{1, \theta} \sin \theta$.

\section{THIRD ORDER SUSCEPTIBILITY WITH FEMTOSECOND PULSES}

Actually, $\rho_{a c}$ is composed of two parts, the linear $\rho_{a c}^{(1)}$ and nonlinear $\rho_{a c}^{(3)}$ parts. Although the size of the spore is small (about $1 \mu \mathrm{m}$ ), the absorption or gain coefficient may be large due to the resonance of the probe. This is characterized by the linear part which is proportional to the anti-Stokes field. It gives the linear response of the medium through dielectric function or linear susceptibility which describes the reflection, refraction and absorption or amplification processes. The nonlinear part gives the third order polarization,

$$
\widetilde{P}_{p}^{(3)}\left(\mathbf{r}^{\prime}, \omega\right)=\sum_{q, r, s \in x, y, z} \varepsilon_{0} \widetilde{\chi}_{p q r s}^{(3)} \widetilde{E}_{1 q}\left(\mathbf{r}^{\prime}, \omega\right) \widetilde{E}_{2 r}^{*}\left(\mathbf{r}^{\prime}, \omega\right) \widetilde{E}_{3 s}\left(\mathbf{r}^{\prime}, \omega\right) .
$$

Using Eqs. (9), (14), and (D2), we have the third order susceptibility

$$
\begin{aligned}
\tilde{\chi}_{p q r s}^{(3)}\left(\mathbf{r}^{\prime}, \omega\right)= & \frac{i \eta \wp_{p, c a} \wp_{s, a b}}{\varepsilon_{0} \hbar\left(\gamma_{a c}-i \Delta_{a c}\right)} \frac{u_{1, r}^{*}\left(\mathbf{r}^{\prime}\right) u_{1, q}\left(\mathbf{r}^{\prime}\right) u_{3, s}\left(\mathbf{r}^{\prime}\right)}{\widetilde{E}_{1 q}\left(\mathbf{r}^{\prime}, \omega\right) \widetilde{E}_{2 r}^{*}\left(\mathbf{r}^{\prime}, \omega\right) \widetilde{E}_{3 s}\left(\mathbf{r}^{\prime}, \omega\right)} \\
& \times\left.\sum_{n=0} \frac{1}{n !} \frac{d^{n} E_{3 s}(t)}{d t^{n}}\right|_{t_{3} m=0} ^{n} a_{m n}\left(t_{3}\right) i^{n-m} \\
& \times \frac{d^{n-m} \bar{\rho}_{b c, q r}\left(\Delta_{a c}\right)}{d \Delta_{a c}^{n-m}} .
\end{aligned}
$$

Equation (15) is the proper expression of susceptibility for femtosecond CARS which includes enhanced quantum coherence and electromagnetic induced transparency [22]. This can be seen by keeping only the zeroth order term, taking $\omega \rightarrow \omega_{a c}$ and neglecting the spatial dependence

$$
\tilde{\chi}_{p q r s}^{(3)}=i \eta^{\wp_{p, c a} \wp_{q, a b} \wp_{r, a b}^{*} \wp_{s, \mathrm{ac}}} \frac{\left(w_{c} \Gamma_{a b}+w_{b} \Gamma_{a c}^{*}\right)}{\varepsilon_{0} \hbar^{3} \gamma_{a c}},
$$

where $Y(0)=\left(\Gamma_{a b} \Gamma_{a c}^{*} \Gamma_{b c}^{*}+\left|\Omega_{1}\right|^{2} \Gamma_{a c}^{*}+\left|\Omega_{2}\right|^{2} \Gamma_{a b}\right)$. Equation (15) reduces to the conventional perturbative result by setting $\left|\Omega_{1,2}\right|^{2}, w_{b} \rightarrow 0$, and $w_{c} \rightarrow-1$. However, it does not take into account the Autler-Townes splitting due to the strong resonant probe field. The exact form of the susceptibility is of course very complex and depends on the chemical composition of the spore, but can be obtained from the experimental results and the transform theory [26]. It would be of interest in spectroscopic studies but need not concern us now.

\section{INTENSITY AND NUMBER OF DETECTED PHOTONS}

Since we are interested in the peak intensity rather than the spectral characteristic of the signal, we set $\omega \rightarrow \omega_{a c}$ hereafter. For CaDPA with tetragonal, hexagonal or trigonal crystal structure, only the diagonal elements of the polarization tensor are nonzero. If the laser fields are polarized along the $x$ axis and keeping only the zeroth order term, Eq. (12) becomes $S(\Theta, \Phi)=i \Sigma_{q} F_{q}(\Theta, \Phi)\left(\wp_{q, a b} / \hbar \gamma_{a c}\right) E_{3 q}(t) \bar{\rho}_{b c, q}(0)$. For isotropic medium Eq. (11), it follows that the ratio of the signal intensity $\mathcal{I}_{4}=\left|\widetilde{\mathbf{E}}_{4}\left(\mathbf{R}, \omega_{a c}\right)\right|^{2}$ to the probe intensity $\mathcal{I}_{3}$ $=\left|\widetilde{\mathbf{E}}_{3}\left(\omega_{a c}\right)\right|^{2}$ takes a simple form

$$
\frac{\mathcal{I}_{4}\left(\mathbf{R}, \omega_{a c}\right)}{\mathcal{I}_{3}\left(\omega_{a c}\right)}=u\left(\frac{3}{8 \pi R} \lambda \frac{\gamma_{r}}{\gamma_{a c}} N\left|\bar{\rho}_{b c}(0)\right| F\right)^{2}
$$

where $u=\left|\hat{\varepsilon}_{4}\right|^{2}, \gamma_{r}=\wp_{c a} \wp_{a b} \omega_{a c}^{3} / 3 \pi \varepsilon_{0} \hbar c^{3}$, and $\lambda=2 \pi c / \omega_{a c}$.

The signal power collected by a detector subtending the angle $\Theta_{1}$ to $\Theta_{2}$ is (Fig. 4)

$$
P_{4}\left(\mathbf{R}, \omega_{a c}\right)=\frac{\varepsilon_{0} c}{2} \int_{\Theta_{1}}^{\Theta_{2}} d \Theta R^{2} \sin \Theta \int_{0}^{2 \pi} d \Phi\left|\widetilde{\mathbf{E}}_{4}\left(\mathbf{R}, \omega_{a c}\right)\right|^{2}
$$

In the far zone and forward direction we integrate from $\Theta_{1}$ $=0$ to $\Theta_{2}=\beta$ and while for backward direction $\Theta_{1}=\pi-\beta$ to $\Theta_{2}=\pi$, where $\tan \beta=d / 2 R$ and $d$ is detector diameter. Since $\widetilde{\mathbf{E}}_{4}\left(\omega_{a c}\right)$ varies insignificantly over this small angular range of $\beta$, it is a good approximation to write the forward $(f)$ and backward $(b)$ powers as

$$
P_{4}^{f(b)}\left(\mathbf{R}, \omega_{a c}\right)=\frac{\varepsilon_{0} c}{2}(1-\cos \beta) R^{2} \int_{0}^{2 \pi}\left|\widetilde{\mathbf{E}}_{4}\left(\mathbf{R}, \omega_{a c}\right)\right|_{\Theta=0(\pi)}^{2} d \Phi
$$

Thus, the ratio of the signal power collected by the detector to the probe laser power $P_{3}\left(\omega_{a c}\right)=\varepsilon_{0}\left|\widetilde{\mathbf{E}}_{3}\left(\omega_{a c}\right)\right|^{2} c A / 2$ is 


$$
\frac{P_{4}^{f(b)}\left(\mathbf{R}, \omega_{a c}\right)}{P_{3}\left(\omega_{a c}\right)}=\frac{(1-\cos \beta) R^{2}}{A} \int_{0}^{2 \pi} \frac{\mathcal{I}_{4}\left(\mathbf{R}, \omega_{a c}\right)_{\Theta=0(\pi)}}{\mathcal{I}_{3}\left(\omega_{a c}\right)} d \Phi,
$$

where $A$ is the beam cross section.

We are interested in the ratio of the backscattered number of photons to the number of probe photons, $n_{4} / n_{3}$. If we use 222a circular probe beam of area $A=\pi w^{2} / 4$ with beam diameter $w$ at the spore, the ratio $n_{4} / n_{3}$ follows from $P_{3}$ $\simeq n_{3} \hbar \nu_{3} / \tau_{3}, P_{4} \simeq n_{4} \hbar \nu_{4} / \tau_{4}, \nu_{4} / \tau_{4} \simeq \nu_{3} / \tau_{3}$ and Eq. (20) as

$$
\frac{n_{4}\left(\mathbf{R}, \omega_{a c}\right)}{n_{3}}=\frac{\epsilon}{2 \pi}\left(\frac{d}{w}\right)^{2} \int_{0}^{2 \pi} \frac{\mathcal{I}_{4}\left(\mathbf{R}, \omega_{a c}\right)_{\Theta=0(\pi)}}{\mathcal{I}_{3}\left(\omega_{a c}\right)} d \Phi,
$$

where $\epsilon$ is the detector efficiency with $\tau_{3}$ and $\tau_{4}$ are the pulse durations of the probe and signal. The ratio $n_{4} / n_{3}$ would be time independent if the coherence $\rho_{b c}(t)$ evolves adiabatically with the probe pulse. Thus, Eqs. (17) and (21) give the simplistic result of Eq. (1).

The aim is to estimate $n_{4}$ the number of photons backscattered into the detector. Given the probe laser pulse of energy $U_{3}=10 \mathrm{~mJ}$, duration $\tau_{3}$ and beam diameter $A$, we compute the probe field intensity $\mathcal{I}_{3}$ from $P_{3}\left(\omega_{a c}\right)=\frac{1}{2} \varepsilon_{0} \mathcal{I}_{3} c A \simeq U_{3} / \tau_{3}$. The backscattered power per pulse excitation $P_{4} \propto n_{4} / \tau_{4}$ $\propto \mathcal{I}_{3} N^{2}$ is proportional to the probe field intensity $\mathcal{I}_{3}$ (not the energy), and also to the square of $N$ (the number of DPA molecules) instead of the number density $\eta=N / V$. The number density of the DPA molecules does not affect the number of detectable photons as long as the cross section of the spore falls within the beam cross section. Only the photons that fall on the spore contribute to the detected signal. Thus, it is the intensity $\mathcal{I}_{3}$ and not the energy $U_{3}$ which determines the amount of detectable signal.

\section{ANALYTICAL RESULTS FOR HOMOGENEOUS INTERNAL FIELDS}

In this section, we obtain analytical results for the scattering efficiency when the incident fields are not refracted by the spore. This approximation applies only to a small particle or long wavelength regime $\rho_{0} / \lambda \ll 1$. Effectively, the incident fields do not undergo focusing effect and there is no internal reflection. An incident laser field, say $x$-polarized, remains essentially homogeneous within the spore. Thus, $u_{f, q}\left(\mathbf{r}^{\prime}\right)$ $\approx \delta_{q x} e^{i\left(\nu_{f} / c\right) \hat{\mathrm{k}}_{f} \mathbf{r}^{\prime}}$ and the third order polarization takes a simple form $\quad \widetilde{P}_{p}^{(3)}\left(\mathbf{r}^{\prime}, \omega_{a c}\right) \simeq \eta \wp_{p, c a} i e^{i \mathbf{k}_{\mathrm{CARS}} \cdot \mathbf{r}^{\prime}}\left(\widetilde{\Omega}_{3} / \gamma_{a c}\right) \bar{\rho}_{b c}\left(\omega_{a c}\right)$. Hence, Eqs. (10), (11), and (13) give

$$
\tilde{\mathbf{E}}_{4}\left(\mathbf{R}, \omega_{a c}\right)=\hat{\varepsilon}_{4} e^{i k_{4}\left(\omega_{a c}\right) R} \frac{\mu_{0} \omega_{\mathrm{ac}}^{2} \wp_{x, c a}}{4 \pi R} \frac{i \widetilde{\Omega}_{3}}{\gamma_{a c}} N \bar{\rho}_{b c}(0) F(\Theta, \Phi)
$$

with

$$
\begin{aligned}
F(\Theta, \Phi)= & \frac{1}{V} \int_{0}^{\rho_{0}} d \rho \rho^{2} \int_{0}^{\pi} d \theta \sin \theta \int_{0}^{2 \pi} d \phi \\
& \times e^{i \mathbf{k}_{\mathrm{CARS}} \cdot \mathbf{r}^{\prime}} e^{-i k_{4}\left(\omega_{a c}\right) \rho[\cos \Theta \cos \theta+\cos (\phi-\Phi) \sin \Theta \sin \theta]}
\end{aligned}
$$

where

$$
\begin{aligned}
\hat{\varepsilon}_{4}= & \sum_{l} \hat{1} W_{l, x}=(\hat{\mathrm{R}} \times \hat{\mathrm{x}}) \times \hat{\mathrm{R}} \\
= & \left(\cos ^{2} \Theta \cos ^{2} \Phi,-\sin ^{2} \Theta \cos \Phi \sin \Phi,\right. \\
& -\sin \Theta \cos \Theta \cos \Phi)
\end{aligned}
$$

is the observation direction for dipoles aligned along $x$ axis and $\mathbf{k}_{\mathrm{CARS}}=\left(\nu_{1} \hat{\mathrm{k}}_{1}-\nu_{2} \hat{\mathrm{k}}_{1}+\nu_{3} \hat{\mathrm{k}}_{3}\right) / c$ is the four-wave mixing wave vector which should be contrasted with the signal (antiStokes) wave vector $k_{4}\left(\omega_{a c}\right)$. Note that we could not write $k_{f}=n_{f} \nu_{f} / c$ which would contradict the assumption of no refractive index effect.

If all the laser fields are collinear and directed along $+z$ direction, the angular factor for spherical spore of radius $\rho_{0}$ depends only on $\Theta$ (see Fig. 4) and Eq. (22) reduces to the two-dimensional integration

$$
\begin{aligned}
F(\Theta)_{\text {sphere }}= & \frac{2 \pi}{V_{\text {sphere }}} \int_{0}^{\rho_{0}} \rho^{2} d \rho \int_{-1}^{1} e^{i \Delta k \rho x} \\
& \times J_{0}\left[\left(n_{4} \omega_{a c} \rho / c\right) \sin \Theta \sqrt{1-x^{2}}\right] d x,
\end{aligned}
$$

where $\Delta k=k_{\mathrm{CARS}}-\left(n_{4} \omega_{a c} / c\right) \cos \Theta$ is the direction dependent phase mismatch, $V_{\text {sphere }}$ is the volume of the sphere and $x$ $=\cos \theta$.

For a cylindrical spore with arbitrary orientation $\vartheta$ (see Fig. 4) and arbitrary observation point $(\Theta, \Phi)$ we obtain an analytical expression

$$
F(\Theta, \Phi, \vartheta)_{\mathrm{cyl}}=\frac{2 J_{1}\left[r_{0} s(\Theta, \Phi, \vartheta)\right]}{r_{0} s(\Theta, \Phi, \vartheta)} \frac{\sin \frac{1}{2} H f(\Theta, \Phi, \vartheta)}{\frac{1}{2} H f(\Theta, \Phi, \vartheta)},
$$

where

$$
f(\Theta, \Phi, \vartheta)=\Delta k \cos \vartheta-\left(n_{4} \nu_{4} / c\right) \sin \Theta \sin \Phi \sin \vartheta,
$$

$$
\begin{aligned}
s(\Theta, \Phi, \vartheta)= & {\left[\left\{\left(n_{4} \nu_{4} / c\right) \sin \Theta \cos \Phi\right\}^{2}\right.} \\
& \left.+\left\{\left(n_{4} \nu_{4} / c\right) \sin \Theta \sin \Phi \cos \vartheta+\Delta k \sin \vartheta\right\}^{2}\right]^{1 / 2}
\end{aligned}
$$

The $\Phi$ dependence is due to azimuthal asymmetry when the cylindrical spore is tilted ( $\vartheta$ finite) relative to the laser direction ( $z$ axis). Of course, $F(\Theta, \Phi, \vartheta)_{\text {cyl }}$ is independent of $\Phi$ if the cylinder is aligned along the $z$ axis $(\vartheta=0)$.

So, Eqs. (24) and (25) give the expressions of $F$ for sphere, cylinder aligned along $(\|)$ detection axis and perpendicular $(\perp)$ to the axis are, respectively,

$$
F_{\text {sphere }}=\frac{3}{\left(\rho_{0} \Delta k^{ \pm}\right)^{2}}\left(\frac{\sin \rho_{0} \Delta k^{ \pm}}{\rho_{0} \Delta k^{ \pm}}-\cos \rho_{0} \Delta k^{ \pm}\right),
$$




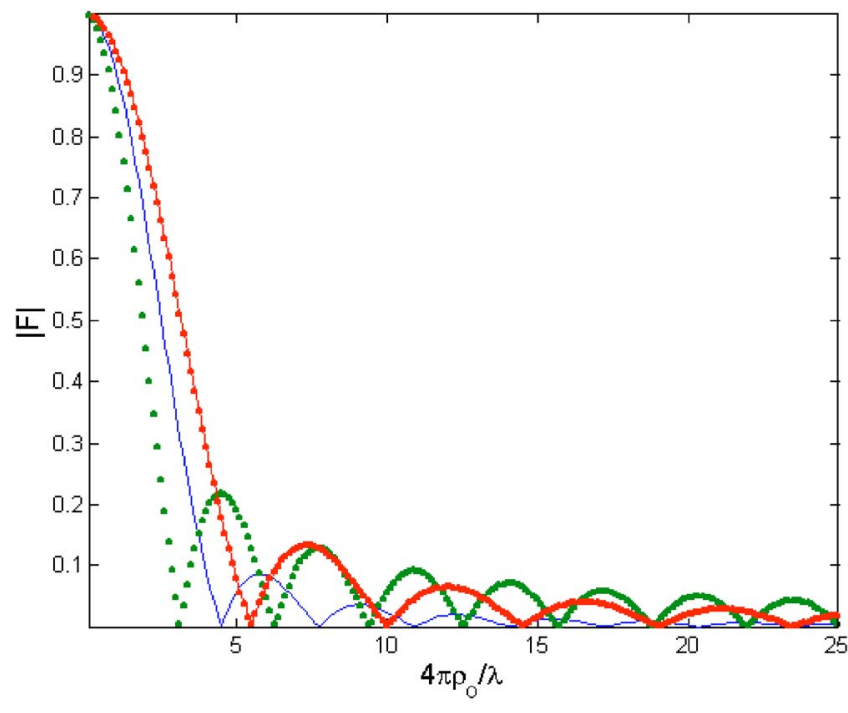

FIG. 5. (Color online) Absolute values of the geometrical factor $F$ as a function of $x$ for three different geometries, (a) sphere (-) $x=\rho_{0}$, (b) cylinder along detection direction $(\cdots) x=H$, and (c) cylinder perpendicular to detection direction (-.--) $x=r_{0}$, computed from Eqs. (29)-(31), respectively. The sphere radius is $\rho_{0}$ $=0.5 \mu \mathrm{m}$, cylinder radius $r_{0}=0.35 \mu \mathrm{m}$, and cylinder length $H$ $=1 \mu \mathrm{m}$.

$$
\begin{gathered}
F_{\text {cyl } \|}=F_{\Theta=\pi, \vartheta=0 \text { or } \pi}=\frac{\sin H \Delta k^{ \pm} / 2}{H \Delta k^{ \pm} / 2}, \\
F_{\text {cyl } \perp}=F_{\Theta=\pi, \vartheta=\pi / 2}=\frac{2 J_{1}\left(r_{0} \Delta k^{ \pm}\right)}{r_{0} \Delta k^{ \pm}},
\end{gathered}
$$

where $\Delta k^{-}=k_{\mathrm{CARS}}-\left(n_{4} \omega_{a c} / c\right)$ and $\Delta k^{+}=k_{\mathrm{CARS}}+\left(n_{4} \omega_{a c} / c\right)$ are corresponding phase mismatch for observation in the forward $\Theta=0$ and backward $\Theta=\pi$ directions, respectively.

As we can see, the factors $F$ defined in Eqs. (28)-(30) depend only on the dimension along the observation direction which varies according to the spore geometry and orientation, as well as on the wave vector mismatch $\Delta k^{ \pm}$, which is inversely proportional to the wavelength $\lambda$. As seen in Fig. 5, a wavelength larger than the spore dimension $x \in \rho_{0}, H$ and $r_{0}$ would correspond to a larger $F$ and larger signal. Also, a small particle generally provides larger signal since the phase matching condition $\lambda \gtrsim x$ is easily fulfilled.

The simple model gives some insights on the roles of geometry and phase matching in the scattering process. The simplicity and elegance of the above analysis are at the expense of some physical effects, like local field and refraction and reflection (lensing) of the incident fields in the microsphere. When these effects are included, the volume integration can only be performed numerically.

\section{DISCUSSIONS}

The results are separated into two sections. (A) Physical explanations and discussions are given for the large backscattered signal in Fig. 3 and the simulated intensity profiles in Fig. 6. (B) Numerical estimates of the number of detectable backscattered photons are given with the potential applications discussed.
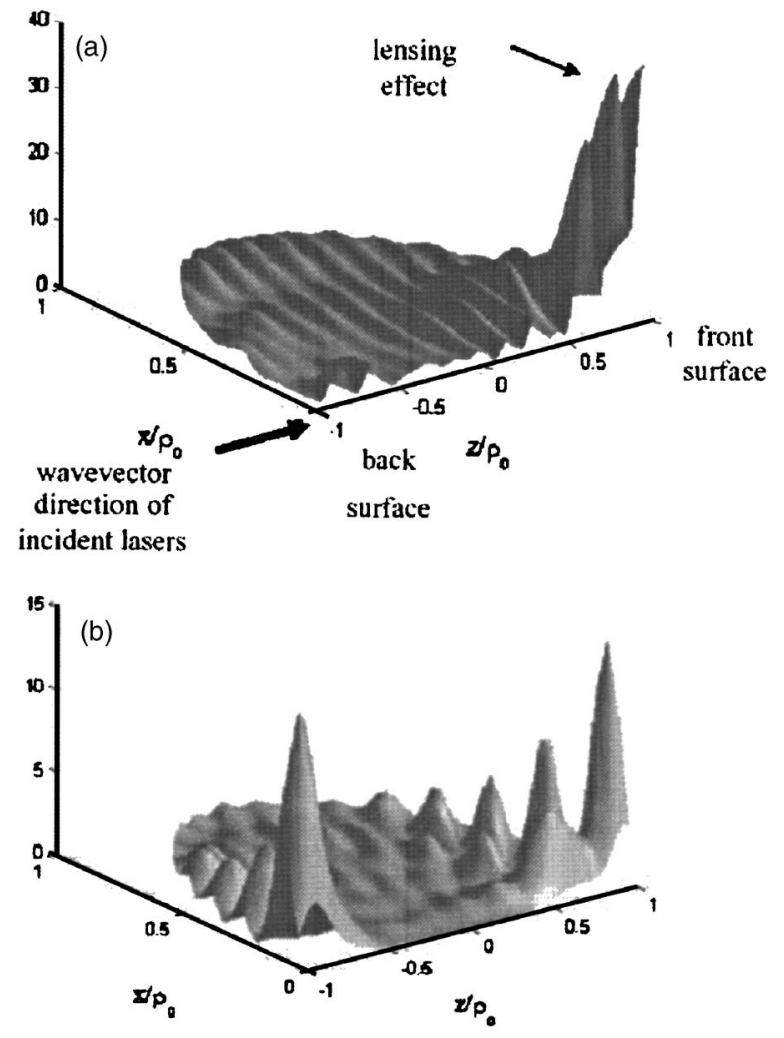

FIG. 6. Normalized intensity distributions of the incident fields in a spherical spore computed from the Lorenz-Mie theory. (a) $x$ component $\left(\left|u_{f, x}\right|^{2}\right)$ and (b) $z$ component $\left(\left|u_{f, z}\right|^{2}\right)$ for $\phi=0$. The incident field $f$ is polarized along the $x$ axis. Note the high intensity of the $x$ component near the front surface.

\section{A. Lensing and refractive effects}

The three incident laser fields undergo focusing (lensing) inside the sphere. The inhomogeneous distributions of the internal fields $\mathbf{E}_{\text {int }}(\rho, \varphi, z, t) \in \mathbf{E}_{1,2,3}(\mathbf{r}, t)$ are obtained numerically from the Lorenz-Mie theory [23] as given in Appendix E. For a $x$-polarized incident field, Fig. 6 shows that the $x$ component of the internal field is strongly focused near the front surface of the spore. However, the $z$ component field is strongly distributed near both the front and back surfaces. The refractive index contrast $\varepsilon$ between the air and the spore leads to multiple internal reflections of the incident fields. Mathematically, the index contrast makes the Mie coefficients $c_{n}$ and $d_{n}$ differ from unity [Eqs. (E4) and (E5)], resulting in the inhomogeneous field distribution and lensing. However, multiple internal reflections and interference also occur in thin film [24] but there is no lensing. Thus, the lensing effect in the spore is a geometrical manifestation of multiple internal reflections.

The results of Fig. 3 have been plotted using Eqs. (13), (17), and (E6)-(E8). The large backscattered signal we obtain should not be attributed directly to lensing. Lensing simply redistributes the field intensity; regions of higher intensity would generate larger CARS signal at the expense of regions of lower intensity. The actual cause of large backscattered signal is backward phase matching of internally reflected laser fields. Multiple internal reflections occur at the 
TABLE I. Parameters of the spore used in our estimation.

\begin{tabular}{ccc}
\hline \hline Number of molecules & $N$ & $5 \times 10^{8}$ \\
\hline Coherence between vibrational levels & $\left|\rho_{b c}\right|$ & 0.5 \\
Radiative decay & $\gamma_{r}$ & $10^{8} \mathrm{~s}^{-1}$ \\
Decoherence rate & $\gamma_{a c}$ & $2.5 \times 10^{13} \mathrm{~s}^{-1}$ \\
Anti-Stokes wavelength & $\lambda$ & $0.250 \mu \mathrm{m}$ \\
Spherical spore radius & $\rho_{0}$ & $0.5 \mu \mathrm{m}$ \\
Cylindrical spore radius & $r_{0}$ & $0.35 \mu \mathrm{m}$ \\
Cylindrical spore length & $H$ & $1 \mu \mathrm{m}$ \\
\hline \hline
\end{tabular}

inner surface of the spore which acts something like a resonant cavity with low reflectance (about 4\%). Inhomogeneous internal fields are created from the interference of infinite spherical wave components $\{n\}$ propagating in all directions, as represented by the associate Legendre polynomials $P_{n 1}(\theta)$ and the fractional Bessel functions $J_{n+1 / 2}(k r)$. For example, $J_{3 / 2}(k r)=\sqrt{2 / \pi k r}[(\sin k r / k r)$ $-\cos k r$ ] contains the forward component $e^{i k r}$ as well as backward component $e^{-i k r}$ through the "sine" and "cosine." Thus, the anti-Stokes coherence, Eq. (D5) in Appendix D also acquires a backward phase factor (underlined), $\tilde{\rho}_{a c}^{(3)}\left(\mathbf{r}^{\prime}, \omega\right) \sim(1-R) e^{i\left(k_{1}-k_{2}+k_{3}\right) z}+\mathcal{R} e^{-i\left(k_{1}-k_{2}+k_{3}\right) z}+\cdots \quad$ [25]. For backscattered signal $\left(\Theta=180^{\circ}\right)$, the integrand in Eq. (11) becomes $\quad(1-R) e^{i\left(k_{4}+k_{1}-k_{2}+k_{3}\right) \rho \cos \theta}+R e^{i\left(k_{4}-k_{1}+k_{2}-k_{3}\right) \rho \cos \theta_{+} \cdot \cdots}$. The first term corresponds to phase mismatch while the second term has phase matching due to the backward (internally reflected) components of the laser fields. Typically, about $4 \%$ of the incident field is internally reflected, resulting in the corresponding large amount of backscattered CARS signal. This is accompanied by some reduction in the forward CARS signal compared to the result for homogeneous field, as shown in Fig. 3. Finally, we note that the generation of CARS signal occurs in the spore upon the input of the three laser fields. Although the process is nonlinear, the large backscattered signal is the result of linear mechanisms of reflection and refraction, backward phase matching and enhanced quantum coherence.

As the ratio of the spore dimension to wavelength decreases, the internal fields become essentially homogeneous within the spore. The angular profile is shown in Fig. 3 (thin curve). The lensing effect vanishes and the scattered field can be well described by the analytical results in Sec. IV. Dispersion, absorption or amplification of the incident field can only be incorporated phenomenologically through the complex dielectric functions $n_{f}$ appearing in Eqs. (E4) and (E5). However, the signal would experience electromagnetically induced transparency [22] as it propagates through the spore because of the strong resonant probe field. This effect is taken into account by the complex linear susceptibility $\chi^{(1)}$ derived in Sec. VI, in addition to the phenomenological refractive index due to background molecules.

\section{B. Estimation of number of detected photons}

In the following, we apply Eqs. (17) and (21) with the parameters in Tables I and II to estimate the forward and
TABLE II. Laser and detection parameters used in our estimation.

\begin{tabular}{ccc}
\hline \hline Distance between detector and spore & $R$ & $1 \mathrm{~km}$ \\
Detector diameter & $d$ & $1 \mathrm{~m}$ \\
Detector efficiency & $\epsilon$ & 0.1 \\
Laser beam diameter at the spore & $w$ & {$[30] 2 \mathrm{~mm}$} \\
\hline \hline
\end{tabular}

backward detection efficiencies of scattered signal from an anthrax spore. The results are summarized in Table III.

Simple analysis without lensing effect based on Eqs. (28)-(30) shows that the backward signal is too weak to be useful. However, when lensing effect is included, the backward signal is more than $10^{4}$ larger, as seen from Fig. 3. It is interesting to note that the backward signal is almost as strong as the forward signal. Although the detectable signal is more than 10 orders of magnitude smaller compared to the probe laser, there are of finite number of photons to be detected within $1 \mathrm{~km}$ distance from the spore. Using a typical laser pulse energy of $U_{3}=10 \mathrm{~mJ}$ which contains $n_{3}$ $=U_{3} / \hbar \nu_{3} \sim 10^{16}$ photons and Eq. (21), we estimate numerically that there is a substantial number (about $10^{4}$ ) of backscattered photons. This encouraging result shows that nonlinear backscattered CARS signal can be very useful for standoff detection of particles.

However, for rapid identification of spores in the atmosphere, sufficiently large number of detected photons is required for precise spectroscopic analysis within a small integration time. The precision of the detected signal can be enhanced by making repeated measurements. This is done by probing the spore using a series of short pulses. Typical repetition rate of up to $r_{p}=1000 \mathrm{pulse} / \mathrm{s}$ can be produced. Hence, if we assume $\mathcal{I}_{4}$ to be independent of $\Phi$ in Eq. (21) the total number of photon counts per second can be expressed as

$$
\begin{aligned}
N_{4} \simeq & r_{p} \epsilon\left(\frac{d}{w}\right)^{2} \frac{\mathcal{I}_{4}}{\mathcal{I}_{3}}=r_{p} \frac{\lambda}{8 \pi Z_{R}} \underbrace{\epsilon \frac{A_{d}}{4 \pi R^{2}}}_{\text {detection efficiency }} \\
& \times \frac{1}{M} \sum_{j=1}^{M} \underbrace{n_{3}\left(N \frac{\gamma_{r}}{\gamma_{a c}} \rho_{b c}(t) 3 F\left(\Theta, \rho_{0 j}\right)\right)^{2}}_{\text {number of backscattered counts per spore }},
\end{aligned}
$$

where $M$ is the number of averaging over various spore

TABLE III. Summary of the ratio of detected number of photons to the number of probe photons $n_{4} / n_{3}$ for forward $(f)$ and backward (b) directions. The absolute number of detected photons are given for a probe pulse containing $10^{16}$ photons.

\begin{tabular}{ccc}
\hline \hline Photon number ratio & Without lensing & With lensing \\
\hline$n_{4}^{f \text { ssphere }} / n_{3}$ & $1.7 \times 10^{-11}$ & $2.9 \times 10^{-12}$ \\
$n_{4}^{b, \text { sphere }} / n_{3}$ & $2.9 \times 10^{-17}$ & $9.7 \times 10^{-13}$ \\
$n_{4}^{b, \text { sphere }} / n_{4}^{f \text {,sphere }}$ & $1.7 \times 10^{-6}$ & 0.3 \\
$n_{4}^{f \text {,sphere }}$ & $2 \times 10^{5}$ & $3.6 \times 10^{4}$ \\
$n_{4}^{b \text {,sphere }}$ & $0-1$ & $1.2 \times 10^{4}$ \\
\hline \hline
\end{tabular}


sizes, $Z_{R} \doteq \pi w^{2} / 2 \lambda$ is the Rayleigh range, and $A_{d}=\pi d^{2} / 4$ is the detector area. It follows that

$$
N_{4}^{b, \text { sphere }}=n_{4}^{b \text {,sphere }} r_{p} \sim 10^{7} \text { counts } / \mathrm{s} .
$$

This is a large number of backscattered photons that can be detected from one spore. Precise spectroscopic data of the spore can be constructed and compared with the standard spectroscopic data. The encouraging numerical result supports the idea of using coherent backscattered CARS signal for standoff detection and identification of spores. Thus, this work would be extended to agglomerate or clumps of spores.

Before we conclude, there is a concern here of how does atmospheric condition such as cloud, air turbulence and temperature fluctuation affect the propagation of the femtosecond laser pulses and the generated CARS signal. The laser power should be at a nondestructive level for probing biological matter, thus plasma formation and filamentation [28] do not occur. The coherence of laser pulses would deteriorate after propagation through a distance in the atmosphere. Pulse distortion and reshaping may occur in addition to diffraction. These effects may be precompensated by properly shaped pulse and employing adaptive optics [29] at the ground telescope before transmission of the pulses. The signal received can be corrected similarly as well. Also, signal processing programs for spectroscopic corrections can be employed. Further studies of atmospheric effects on the spectroscopic contents of the signal and ways to obtain reliable signal will be reported elsewhere. This would strengthen the foundation of this technique for applications in many areas of remote analysis of airborne chemicals based on quantum coherence and femtosecond pulses.

\section{CONCLUSIONS}

We have presented a detailed semiclassical theory to describe and study the backscattering of femtosecond CARS signal from a microparticle or spore. For the first time we incorporate the Lorenz-Mie theory and femtosecond dynamics in the nonlinear process. The four-wave mixing signal produced is enhanced via quantum coherence. The theory gives encouraging result. There is a large number of coherently scattered photons in the backward direction. This is within the detection ability of conventional techniques and therefore should be the motivation for further experimental works, particularly in spectroscopic characterization of anthrax spore using backscattered signal and ultimately for standoff detection of clumps of spores in the atmosphere. We have also derived expressions for the Raman coherence, antiStokes coherence and the nonlinear susceptibility beyond the perturbative and steady state regimes. These results are valid for strong and short pulses and should serve as a good basis for further studies of the ultrafast quantum dynamics using femtosecond lasers in atomic and molecular systems.

\section{ACKNOWLEDGMENTS}

We gratefully acknowledge the support from the Air Force Office of Scientific Research, the Office of Naval Research (Grant No. N00014-02-1-0478), the Air Force Re- search Laboratory (Rome, NY), Defense Advanced Research Projects Agency, and the Robert A. Welch Foundation (Grant No. A-1261). Y.R. thanks C. Hovde, A. Muthukrishnan, and Z.E. Sariyanni for useful and fruitful discussions.

\section{APPENDIX A: DISCRETE EXPRESSION OF ELECTRIC FIELD}

Using $\mathbf{P}\left(\mathbf{r}^{\prime}, t^{\prime}\right)=\sum_{j} \hat{\mathrm{d}}_{j} d_{j} \delta\left(\left|\mathbf{R}-\mathbf{r}^{\prime}\right|-\left|\mathbf{R}-\mathbf{r}_{j}\right|\right)$, we convert Eq. (3) to a discrete form

$$
\widetilde{\mathbf{E}}_{4}(\mathbf{R}, \omega)=\left(\boldsymbol{\nabla} \boldsymbol{\nabla} \cdot+\frac{\omega^{2}}{c^{2}}\right) \sum_{j} \hat{\mathrm{d}}_{j} d_{j}(\omega) \frac{e^{i \omega \mid \mathbf{R}-\mathbf{r}_{j} / c}}{4 \pi \varepsilon_{0}\left|\mathbf{R}-\mathbf{r}_{j}\right|},
$$

where $d_{j}(\omega)=\operatorname{Tr}\left\{\hat{\wp}_{j} \hat{\rho}(\omega)\right\}$ is the mean dipole moment of the $j$ th molecule. We use $\left|\mathbf{R}-\mathbf{r}_{j}\right| \simeq R-\hat{\mathbf{R}} \cdot \mathbf{r}_{j}$ for far field to evaluate $\boldsymbol{\nabla} \cdot\left[\left(\hat{d}_{j} / R\right) e^{i \omega\left(R-\hat{\mathrm{R}} \cdot \mathbf{r}_{j}\right) / c}\right] \simeq \hat{\mathbf{R}} \cdot \hat{\mathrm{d}}_{j} i(\omega / c)\left(e^{i \omega\left(R-\hat{\mathrm{R}} \cdot \mathbf{r}_{j}\right) / c} / R\right)$ since in far field, $(\omega / c) R \gg 1$. Hence, we have evaluated $\boldsymbol{\nabla} \boldsymbol{\nabla} \cdot\left[\left(\hat{d}_{j} / R\right) e^{i \omega\left(R-\hat{\mathrm{R}} \cdot \mathbf{r}_{j}\right) / c}\right]=-\hat{\mathrm{R}}\left(\hat{\mathrm{R}} \cdot \hat{\mathrm{d}}_{j}\right)(\omega / c)^{2}\left(e^{i \omega\left(R-\hat{\mathrm{R}} \cdot \mathbf{r}_{j}\right) / c} / R\right)$.

By using $\hat{\mathrm{d}}_{j}-\hat{\mathrm{R}}\left(\hat{\mathrm{R}} \cdot \hat{\mathrm{d}}_{j}\right)=\left(\hat{\mathrm{R}} \times \hat{\mathrm{d}}_{j}\right) \times \hat{\mathrm{R}}$ we obtain the discrete form of the signal field

$$
\widetilde{\mathbf{E}}_{4}(\mathbf{R}, \omega)=\frac{\omega^{2}}{c^{2}} \sum_{j}\left[\left(\hat{\mathrm{R}} \times \hat{\mathrm{d}}_{j}\right) \times \hat{\mathrm{R}}\right] d_{j}(\omega) \frac{e^{i \omega\left(R-\hat{\mathrm{R}} \cdot \mathbf{r}_{j}\right) / c}}{4 \pi \varepsilon_{0} R} .
$$

The inverse Fourier transform of Eq. (A2) gives

$$
\widetilde{\mathbf{E}}_{4}(\mathbf{R}, t)=-\frac{\mu_{0}}{4 \pi R} \sum_{j}\left[\left(\hat{\mathrm{R}} \times \hat{\mathrm{d}}_{j}\right) \times \hat{\mathrm{R}}\right] \frac{\partial^{2} d_{j}(\tau)}{\partial t^{2}},
$$

where $\tau=t-\left(R-\hat{\mathrm{R}} \cdot \mathbf{r}_{j}\right) / c$ is the retarded time. Using $d_{j}\left(\mathbf{r}_{j}, \tau\right)=\wp_{j, c a} \rho_{a c},\left(\mathbf{r}_{j}, \tau\right)=\wp_{j, c a} \bar{\rho}_{a c},\left(\mathbf{r}_{j}, \tau\right) e^{-i \omega_{a c} \tau}$ we have [21]

$$
\mathbf{E}_{4}(\mathbf{R}, t)=\frac{\mu_{0} \omega_{\mathrm{ac}}^{2}}{4 \pi R} \sum_{j} d_{j}\left(\mathbf{r}_{j}, \tau\right)\left(\hat{\mathrm{R}} \times \hat{\mathrm{d}}_{j}\right) \times \hat{R} e^{-i \omega_{a c}\left(t-\left(R-\hat{\mathrm{R}} \cdot \mathbf{r}_{j}\right) / c\right)},
$$

where $\wp_{j, c a}$ is the dipole matrix element of the $j$ th molecule.

\section{APPENDIX B: DENSITY MATRIX EQUATIONS}

The equations of motion for the elements of the density operator in interaction picture $\left(\bar{\rho}_{x y}\right)$ for a three-level atomic system is written as

$$
\frac{d}{d t} \bar{\rho}_{a a}=\left(i \Omega_{14} \bar{\rho}_{c a}+i \Omega_{23} \bar{\rho}_{b a}+\text { c.c. }\right)-\left(\gamma_{b}+\gamma_{c}\right) \bar{\rho}_{a a},
$$

$$
\begin{aligned}
& \frac{d}{d t} \bar{\rho}_{b b}=\left(-i \Omega_{23} \bar{\rho}_{b a}+\text { c.c. }\right)+\gamma_{b} \bar{\rho}_{a a}-\gamma_{b c}^{r} \bar{\rho}_{b b}, \\
& \frac{d}{d t} \bar{\rho}_{c c}=\left\{-i \Omega_{14} \bar{\rho}_{c a}+\text { c.c. }\right\}+\gamma_{c} \bar{\rho}_{a a}+\gamma_{b c}^{r} \bar{\rho}_{b b},
\end{aligned}
$$




$$
\begin{gathered}
\frac{d}{d t} \bar{\rho}_{a c}=-\gamma_{a c} \bar{\rho}_{a c}+i \Omega_{23} \bar{\rho}_{b c}+i \Omega_{14}\left(\bar{\rho}_{c c}-\bar{\rho}_{a a}\right), \\
\frac{d}{d t} \bar{\rho}_{a b}=-\gamma_{a b} \bar{\rho}_{a b}+i \Omega_{23}\left(\bar{\rho}_{b b}-\bar{\rho}_{a a}\right)+i \Omega_{14} \bar{\rho}_{c b}, \\
\frac{d}{d t} \bar{\rho}_{b c}=-\gamma_{b c} \bar{\rho}_{b c}+i \Omega_{23}^{*} \bar{\rho}_{\mathrm{ac}}-i \Omega_{14} \bar{\rho}_{b a},
\end{gathered}
$$

where $\Omega_{23}=\Omega_{2}(\mathbf{r}, t) e^{-i \Delta_{2} t}+\Omega_{3}(\mathbf{r}, t) e^{-i \Delta_{3} t}, \quad \Omega_{14}=\Omega_{1}(\mathbf{r}, t) e^{-i \Delta_{1} t}$ $+e^{-i \Delta_{4} t} \mathcal{F}_{4}(\mathbf{r}, t) \quad$ with the Rabi frequencies $\Omega_{1}(\mathbf{r}, t)$ $=\Sigma_{q}\left(\mathbf{d} \cdot \hat{\mathbf{e}}_{q}^{*}\right)_{a c} E_{1 q}(\mathbf{r}, t) e^{i \mathbf{k}_{1} \cdot \mathbf{r}}, \quad \Omega_{2,3}(\mathbf{r}, t)=\Sigma_{q}\left(\mathbf{d} \cdot \hat{\mathbf{e}}_{q}^{*}\right)_{a b} E_{2,3 q}(\mathbf{r}, t)$, and $\mathcal{F}=\Sigma_{q}\left(\mathbf{d} \cdot \hat{\mathbf{e}}_{q}^{*}\right)_{a c} E_{4 q}(\mathbf{r}, t)$. The detunings of the four fields are $\Delta_{1}=\nu_{1}-\omega_{\mathrm{ac}}, \Delta_{2}=\nu_{2}-\omega_{a b}, \Delta_{3}=\nu_{3}-\omega_{a b}$, and $\Delta_{4}=\nu_{4}-\omega_{a c}$. The matrix elements $\bar{\rho}_{j i}$ that are slowly time varying, are in the interaction picture, related to that in the Schrödinger picture $\rho_{j i}=\bar{\rho}_{j i} e^{-i \omega_{j i} t}$.

\section{APPENDIX C: GROUND STATE COHERENCE WITH FEMTOSECOND PULSES}

The Raman coherence between $b$ and $c$ is generated by two laser fields $\Omega_{1}$ (pump) and $\Omega_{2}$ (Stokes) with the third (probe) field $\Omega_{3} \simeq 0$. By defining the slowly varying elements as $\pi_{a^{\prime} c}=\bar{\rho}_{a^{\prime} c} e^{i \Delta_{1} t}=\rho_{a^{\prime} c} e^{i \nu_{1} t}, \pi_{b a^{\prime}}=\bar{\rho}_{b a^{\prime}} e^{-i \Delta_{2} t}=\rho_{b a^{\prime}} e^{-i \nu_{2} t}$, and $\pi_{b c}=\bar{\rho}_{b c} e^{-i \Delta_{2} t} e^{i \Delta_{1} t}=\rho_{b c} e^{i\left(\nu_{1}-\nu_{2}\right) t}$, Eqs. (B4)-(B6) are rewritten as

$$
\begin{aligned}
& \frac{d}{d t} \pi_{a^{\prime} c} \simeq-\Gamma_{a^{\prime} c}^{*} \pi_{a^{\prime} c}+i \Omega_{2} \pi_{b c}-i \Omega_{1} w_{c}, \\
& \frac{d}{d t} \pi_{b a^{\prime}} \simeq-\Gamma_{a^{\prime} b} \pi_{b a^{\prime}}+i \Omega_{2}^{*} w_{b}-i \Omega_{1}^{*} \pi_{b c}, \\
& \frac{d}{d t} \pi_{b c} \simeq-\Gamma_{b c}^{*} \pi_{b c}+i \Omega_{2}^{*} \pi_{a^{\prime} c}-i \Omega_{1} \pi_{b a^{\prime}},
\end{aligned}
$$

where the complex decays are defined as $\Gamma_{a^{\prime} c}=\gamma_{a^{\prime} c}+i \Delta_{1}$, $\Gamma_{a^{\prime} b}=\gamma_{a^{\prime} b}+i \Delta_{2}, \quad \Gamma_{b c}=\gamma_{b c}+i\left(\Delta_{2}-\Delta_{1}\right)$ with the inversions $w_{b b}=\bar{\rho}_{a^{\prime} a^{\prime}}-\bar{\rho}_{b b}$ and $w_{c c}=\bar{\rho}_{a^{\prime} a^{\prime}}-\bar{\rho}_{c c}$.

Both fields $\Omega_{2}$ and $\Omega_{1}$ are strong and perturbative solutions do not hold. Steady state solutions are not valid for femtosecond pulses. Assume a two-photon resonance $\Delta_{1}$ $=\Delta_{2}=\Delta$ and $\Omega_{3} \simeq 0$ at the coherence preparation stage. By using the expansion of

$$
\Omega_{i}\left(\mathbf{r}, t^{\prime}\right)=\left.\sum_{n=0} \frac{\left(t^{\prime}-t\right)^{n}}{n !} \frac{d^{n} \Omega_{i}(\mathbf{r}, t)}{d t^{n}}\right|_{t=t_{i}}
$$

around the pulse peak time $t_{i}$ with the substitution $\{i t\}^{m} f(t)$ $\rightarrow\left(d^{m} / d \omega^{m}\right) F(\omega)$, the Fourier transforms of Eqs. (C1) and (C2) can be written as

$$
\begin{aligned}
\pi_{a^{\prime} c}(\mathbf{r}, \omega) \simeq & \frac{1}{\Gamma_{a^{\prime} c}^{*}(\omega)}\left(-i \Omega_{1}(\omega) w_{c c}^{0}+\left.i \sum_{n=0} \frac{1}{n !} \frac{d^{n} \Omega_{2}}{d t^{n}}\right|_{t_{2}}\right. \\
& \left.\times \sum_{m=0}^{n} a_{m n}\left(t_{2}\right) i^{m} \frac{d^{m} \pi_{b c}(\mathbf{r}, \omega)}{d \omega^{m}}\right)
\end{aligned}
$$

$$
\begin{aligned}
\pi_{b a^{\prime}}(\mathbf{r}, \omega) \simeq & \frac{1}{\Gamma_{a^{\prime} b}(\omega)}\left(i \Omega_{2}^{*}(\omega) w_{b b}^{0}-\left.i \sum_{n=0} \frac{1}{n !} \frac{d^{n} \Omega_{1}^{*}}{d t^{n}}\right|_{t_{1}}\right. \\
& \left.\times \sum_{m=0}^{n} a_{m n}\left(t_{1}\right) i^{m} \frac{d^{m} \pi_{b c}(\mathbf{r}, \omega)}{d \omega^{m}}\right),
\end{aligned}
$$

where $w_{c c(b b)}^{0}$ are the steady state values and $\Gamma_{x y}(\omega)=\Gamma_{x y}$ $-i \omega$. Replacing Eqs. (C4) and (C5) into the Fourier transform of Eq. (C3) gives

$$
\bar{\rho}_{b c}(\mathbf{r}, \omega)=\sum_{q, r} \bar{\rho}_{b c, q r}(\omega) u_{1, r}^{*}(\mathbf{r}) u_{1, q}(\mathbf{r})
$$

with the spectral dependence part

$$
\begin{aligned}
\bar{\rho}_{b c, q r}(\omega)= & \frac{\wp_{r, a c}^{*} \wp_{q, a c}}{\hbar^{2}\left[\Gamma_{b c}^{*}(\omega)+\delta_{S}(\omega)\right]_{n=0}} \sum_{m=0}^{n} \frac{i^{n-m}}{n !} \\
& \times\left[\left.w_{c c}^{0} a_{m n}\left(t_{2}\right) \frac{d^{n} E_{2, r}^{*}(t)}{d t^{n}}\right|_{t_{2}} \frac{d^{n-m}}{d \omega^{n-m}}\right. \\
& \times\left(\frac{\tilde{E}_{1, q}(\omega)}{\Gamma_{a^{\prime} c}^{*}(\omega)}\right)+w_{b b}^{0} a_{m n}\left(t_{1}\right) \\
& \left.\times\left.\frac{d^{n} E_{1, q}(t)}{d t^{n}}\right|_{t_{1}} \frac{d^{n-m}}{d \omega^{n-m}}\left(\frac{\widetilde{E}_{2, r}^{*}(\omega)}{\Gamma_{a^{\prime} b}(\omega)}\right)\right],
\end{aligned}
$$

where

$$
\delta_{S}(\omega)=\frac{\left|\Omega_{2}\right|^{2}}{\Gamma_{a^{\prime} c}^{*}(\omega)}+\frac{\left|\Omega_{1}\right|^{2}}{\Gamma_{a^{\prime} b}(\omega)}
$$

is the ac Stark shift and $a_{m n}\left(t_{3}\right)=[n ! / m !(n-m) !]\left(-t_{3}\right)^{m}$. We have written $\Omega_{1}(\mathbf{r}, t)=\Sigma_{q} u_{1, q}(\mathbf{r}) E_{1, q}(t) \wp_{q, a c} / \hbar$ and $\Omega_{2}(\mathbf{r}, t)$ $=\Sigma_{r} u_{1, r}(\mathbf{r}) E_{1, r}(t) \wp_{r, a c} / \hbar$. In order to take into account the spatial inhomogeneity of the laser fields in the particle, we have defined the dimensionless and normalized spatial function $u_{f, q}(\mathbf{r})=\widetilde{E}_{f, q}(\mathbf{r}, \omega) / E_{f}(\omega)$ with $f=1,2,3$ and $q=x, y$.

The coherence for monochromatic fields corresponds to keeping only the zeroth order term in Eq. (C7),

$$
\bar{\rho}_{b c}(\mathbf{r}, \omega) \simeq \frac{\Omega_{1}(\mathbf{r}) \Omega_{2}^{*}(\mathbf{r})}{\left[\Gamma_{b c}^{*}(\omega)+\delta_{S}(\omega)\right]}\left(\frac{w_{c c}^{0}}{\Gamma_{a^{\prime} c}^{*}(\omega)}+\frac{w_{b b}^{0}}{\Gamma_{a^{\prime} b}(\omega)}\right) .
$$

The steady state solution is obtained by setting $\omega \rightarrow 0$. If the Raman fields are not reflected and remain essentially as plane waves within the spore, the spatial dependent part of the fields is entirely contained in the exponential as $\Omega_{1,2}(\mathbf{r})$ $=\Omega_{3} e^{i k_{1,2} z}$ and we may write

$$
\bar{\rho}_{b c}(\mathbf{r}, \omega) \simeq \bar{\rho}_{b c}(\omega) e^{i\left(k_{1}-k_{2}\right) z} .
$$

\section{APPENDIX D: ANTI-STOKES COHERENCE WITH FEMTOSECOND PULSES}

For a probe field $\Omega_{3}$ at resonance, the density matrix element corresponding to the antiStokes coherence Eq. (B4) can be written as 


$$
\bar{\rho}_{a c}^{(3)}(\mathbf{r}, t)=\int_{0}^{t} i \Omega_{3}\left(\mathbf{r}, t^{\prime}\right) \bar{\rho}_{b c}\left(\mathbf{r}, t^{\prime}\right) e^{-\gamma_{a c}\left(t-t^{\prime}\right)} d t^{\prime},
$$

where $\gamma_{a c}$ is the decoherence rate. Similarly, by using the expansion of

$$
\begin{aligned}
\Omega_{3}\left(\mathbf{r}, t^{\prime}\right) & =\left.\sum_{n=0} \frac{\left(t^{\prime}-t\right)^{n}}{n !} \frac{d^{n} \Omega_{3}(\mathbf{r}, t)}{d t^{n}}\right|_{t=t_{3}} \\
& =\sum_{s} u_{3, s}(\mathbf{r}) E_{3 s}\left(t^{\prime}\right) \wp_{s, a b} / \hbar
\end{aligned}
$$

around $t_{3}$, the Fourier transform of the anti-Stokes coherence $\rho_{a c}(\mathbf{r}, t)=\bar{\rho}_{a c}(\mathbf{r}, t) e^{-i \omega_{a c} t}$ can be evaluated exactly

$$
\begin{aligned}
\widetilde{\rho}_{a c}^{(3)}(\mathbf{r}, \omega)= & \left.i \sum_{n=0} \frac{1}{n !} \sum_{s} \frac{u_{3, s}(\mathbf{r})_{\wp, a b}}{\hbar\left(\gamma_{a c}-i \Delta_{a c}\right)} \frac{d^{n} E_{3 s}(t)}{d t^{n}}\right|_{t_{3}} \\
& \times \sum_{m=0}^{n} a_{m n}\left(t_{3}\right) i^{n-m} \frac{d^{n-m} \bar{\rho}_{b c}\left(\mathbf{r}, \Delta_{a c}\right)}{d \Delta_{a c}^{n-m}}
\end{aligned}
$$

where the expression for the ground state coherence $\bar{\rho}_{b c}\left(\mathbf{r}, \Delta_{a c}\right)$ is given in Appendix $\mathrm{C}, \Delta_{a c}=\omega-\omega_{a c}$ and $a_{m n}\left(t_{3}\right)=[n ! / m !(n-m) !]\left(-t_{3}\right)^{m}$. Please note that Eq. (D2) is applicable to arbitrarily short probe pulse. It has the Lorentzian profile with a peak at $\omega=\omega_{a c}$ and FWHM of $2 \gamma_{a c}$.

By inserting $\bar{\rho}_{b c}$ from Eq. (C6) into Eq. (D2) the antiStokes coherence can be written as

$$
\begin{aligned}
\tilde{\rho}_{a c}^{(3)}(\mathbf{r}, \omega)= & i \sum_{q, r, s} u_{1, r}^{*}(\mathbf{r}) u_{1, q}(\mathbf{r}) u_{3, s}(\mathbf{r}) \frac{\wp_{s, a b}}{\hbar\left(\gamma_{a c}-i \Delta_{a c}\right)} \\
& \times\left.\sum_{n=0} \frac{1}{n !} \frac{d^{n} E_{3 s}(t)}{d t^{n}}\right|_{t_{3}} \\
& \times \sum_{m=0}^{n} a_{m n}\left(t_{3}\right) i^{n-m} \frac{d^{n-m} \bar{\rho}_{b c, q r}\left(\Delta_{a c}\right)}{d \Delta_{a c}^{n-m}},
\end{aligned}
$$

where $\bar{\rho}_{b c, q r}\left(\Delta_{a c}\right)$ is given by Eq. (C7). Thus, if the spectral profile of the vibrational coherence is known, the signal coherence can be computed for arbitrary shape of probe pulse.

For quasi-monochromatic fields, the terms with first and higher order derivatives in Eq. (D3) can be neglected and we may rewrite

$$
\widetilde{\rho}_{a c}^{(3)}(\mathbf{r}, \omega)=i \frac{\Omega_{3}(\mathbf{r})}{\hbar\left(\gamma_{a c}-i \Delta_{a c}\right)} \bar{\rho}_{b c}\left(\mathbf{r}, \Delta_{a c}\right) .
$$

If reflection of the Raman and probe fields are neglected, we have

$$
\widetilde{\rho}_{a c}^{(3)}(z, \omega)=\frac{i \Omega_{1} \Omega_{2}^{*} \Omega_{3} e^{i\left(k_{1}-k_{2}+k_{3}\right) z}}{\hbar\left(\gamma_{a c}-i \Delta_{a c}\right) Y(\omega)}\left[w_{c c}^{0} \Gamma_{a^{\prime} b}(\omega)+w_{b b}^{0} \Gamma_{a^{\prime} c}^{*}(\omega)\right],
$$

where

$Y(\omega)=\Gamma_{b c}^{*}(\omega) \Gamma_{a^{\prime} c}^{*}(\omega) \Gamma_{a^{\prime} b}(\omega)+\left|\Omega_{2}\right|^{2} \Gamma_{a^{\prime} b}(\omega)+\left|\Omega_{1}\right|^{2} \Gamma_{a^{\prime} c}^{*}(\omega)$.

\section{APPENDIX E: INCIDENT FIELDS INSIDE THE SPORE}

For an incident plane wave polarized along $x$ axis $\widetilde{\mathbf{E}}_{f, i n}(\mathbf{r}, \omega)=\widetilde{E}_{f 0}(\omega) \hat{\mathbf{x}} e^{i k_{f} z}$, the vector electric field for $f \in 1,2$, 3 inside a linear and homogeneous dielectric medium is given by [23]

$$
\widetilde{\mathbf{E}}_{f}(\mathbf{r}, \omega)=\widetilde{E}_{f 0}(\omega) \sum_{n=1}^{\infty} A_{n}\left(c_{n} \mathbf{M}_{01 n}^{(1)}-i d_{n} \mathbf{N}_{e 1 n}^{(1)}\right)
$$

where

$$
\begin{aligned}
\mathbf{M}_{01 n}^{e(1)}= & \frac{\psi_{n}\left(k_{f} r\right)}{k_{f} r}\left[\mp \hat{\theta} \frac{P_{n 1}(\mu)}{\sin \theta}\left(\begin{array}{c}
\sin \phi \\
\cos \phi
\end{array}\right)-\hat{\phi} \frac{d P_{n 1}(\mu)}{d \theta}\left(\begin{array}{c}
\cos \phi \\
\sin \phi
\end{array}\right)\right], \\
\mathbf{N}_{01 n}^{e(1)}= & \hat{r} \frac{\psi_{n}\left(k_{f} r\right)}{\left(k_{f} r\right)^{2}} n(n+1) P_{n 1}(\mu)\left(\begin{array}{c}
\cos \phi \\
\sin \phi
\end{array}\right)+\frac{1}{k_{f} r} \frac{d \psi_{n}\left(k_{f} r\right)}{d\left(k_{f} r\right)} \\
& \times\left[\hat{\theta} \frac{d P_{n 1}(\mu)}{d \theta}\left(\begin{array}{c}
\cos \phi \\
\sin \phi
\end{array}\right) \mp \hat{\phi} \frac{P_{n 1}(\mu)}{\sin \theta}\left(\begin{array}{c}
\sin \phi \\
\cos \phi
\end{array}\right)\right]
\end{aligned}
$$

with $A_{n}=i^{n}[(2 n+1) / n(n+1)], \mu=\cos \theta$ and $k_{f}=\sqrt{\varepsilon_{f}} \nu_{f} / c$. The coefficients are

$$
\begin{aligned}
c_{f n} & =\sqrt{\varepsilon_{f}} \frac{\psi_{n}\left(a_{f}\right) \xi_{n}^{\prime}\left(a_{f}\right)-\xi_{n}\left(a_{f}\right) \psi_{n}^{\prime}\left(a_{f}\right)}{\psi_{n}\left(\sqrt{\varepsilon_{f}} a_{f}\right) \xi_{n}^{\prime}\left(a_{f}\right)-\sqrt{\varepsilon_{f}} \xi_{n}\left(a_{f}\right) \psi_{n}^{\prime}\left(\sqrt{\varepsilon_{f}} a_{f}\right)} \\
& =\frac{j_{n}\left(a_{f}\right)\left[a_{f} h_{n}\left(a_{f}\right)\right]^{\prime}-h_{n}\left(a_{f}\right)\left[a_{f} j_{n}\left(a_{f}\right)\right]^{\prime}}{j_{n}\left(\sqrt{\varepsilon_{f}} a_{f}\right)\left[a_{f} h_{n}\left(a_{f}\right)\right]^{\prime}-h_{n}\left(a_{f}\right)\left[\sqrt{\varepsilon_{f}} a_{f} j_{n}\left(\sqrt{\varepsilon_{f}} a_{f}\right)\right]^{\prime}},
\end{aligned}
$$

$$
\begin{aligned}
d_{f n} & =\sqrt{\varepsilon_{f}} \frac{\psi_{n}\left(a_{f}\right) \xi_{n}^{\prime}\left(a_{f}\right)-\xi_{n}\left(a_{f}\right) \psi_{n}^{\prime}\left(a_{f}\right)}{\sqrt{\varepsilon_{f}} \psi_{n}\left(\sqrt{\varepsilon_{f}} a_{f}\right) \xi^{\prime}{ }_{n}\left(a_{f}\right)-\xi_{n}\left(a_{f}\right) \psi^{\prime}{ }_{n}\left(\sqrt{\varepsilon_{f}} a_{f}\right)} \\
& =\frac{\sqrt{\varepsilon_{f}}\left\{j_{n}\left(a_{f}\right)\left[a_{f} h_{n}\left(a_{f}\right)\right]^{\prime}-h_{n}\left(a_{f}\right)\left[a_{f} j_{n}\left(a_{f}\right)\right]^{\prime}\right\}}{\varepsilon_{f} j_{n}\left(\sqrt{\varepsilon_{f}} a_{f}\right)\left[a_{f} h_{n}\left(a_{f}\right)\right]^{\prime}-h_{n}\left(a_{f}\right)\left[\sqrt{\varepsilon_{f}} a_{f} j_{n}\left(\sqrt{\varepsilon_{f}} a_{f}\right)\right]^{\prime}},
\end{aligned}
$$

where the prime indicates derivative, $a_{f}=k_{f 0} \rho_{0}, \quad \psi_{n}(x)$ $=\sqrt{\pi x / 2} J_{n+1 / 2}(x)=x j_{n}(x), \xi_{n}(x)=\sqrt{\pi x / 2} H_{n+1 / 2}(x)=x h_{n}(x)$ and $H_{v}(x)=(1 / i \sin \pi v)\left[J_{-v}(x)-e^{-i \pi v} J_{v}(x)\right]=J_{v}(x)+i Y_{v}(x)$ (Hankel first kind) $\left[J_{v}(x)-i Y_{v}(x)\right.$ is the Hankel function of the second kind, $Y_{v}(x)$ is the Bessel function of the second kind or Neumann function]. Also note that $\psi_{n}(a) \xi_{n}^{\prime}(a)$ $-\xi_{n}(a) \psi_{n}^{\prime}(a)=i$. The spherical components are

$$
\widetilde{E}_{f r}=-\frac{\widetilde{E}_{f 0}(\omega)}{\left(k_{f} r\right)^{2}} \sum_{n=1}^{\infty} i A_{n} n(n+1) d_{f n} \psi_{n}\left(k_{f} r\right) P_{n 1}(\mu) \cos \phi,
$$




$$
\begin{aligned}
\widetilde{E}_{f \theta}= & \frac{\widetilde{E}_{f 0}(\omega)}{k_{f} r} \sum_{n=1}^{\infty} A_{n}\left(c_{f n} \psi_{n}\left(k_{f} r\right) \frac{P_{n 1}(\mu)}{\sin \theta}\right. \\
& \left.-i d_{f n} \frac{d \psi_{n}\left(k_{f} r\right)}{d\left(k_{f} r\right)} \frac{d P_{n 1}(\mu)}{d \theta}\right) \cos \phi, \\
\widetilde{E}_{f \phi}= & \frac{\tilde{E}_{f 0}(\omega)}{k_{f} r} \sum_{n=1}^{\infty} A_{n}\left(-c_{f n} \psi_{n}\left(k_{f} r\right) \frac{d P_{n 1}(\mu)}{d \theta}\right. \\
& \left.-i d_{f n} \frac{d \psi_{n}\left(k_{f} r\right)}{d\left(k_{f} r\right)} \frac{P_{n 1}(\mu)}{\sin \theta}\right) \sin \phi .
\end{aligned}
$$

We use the recurrence relations in numerical computations,

$$
\frac{d P_{n m}(\mu)}{d \theta}=-(n+1) \mu \frac{P_{n m}(\mu)}{\sin \theta}+(n-m+1) \frac{P_{n+1, m}(\mu)}{\sin \theta}
$$

from p. 365 in Ref. [27],

$$
\begin{aligned}
\frac{d \psi_{n}(x)}{d x}= & \frac{1}{4} \sqrt{\frac{2 \pi}{x}} J_{n+(1 / 2)}(x)+\frac{1}{2} \sqrt{\frac{\pi x}{2}}\left[J_{n-(1 / 2)}(x)-J_{n+(3 / 2)}\right. \\
& \times(x)],
\end{aligned}
$$

since $d J_{v}(x) / d x=\left[J_{v-1}(x)-J_{v+1}(x)\right]$ (p. 586 in Ref. [31]) and

$$
\begin{aligned}
\frac{d \xi_{n}(x)}{d x}= & \frac{1}{4} \sqrt{\frac{2 \pi}{x}} H_{n+(1 / 2)}(x)+\frac{1}{2} \sqrt{\frac{\pi x}{2}}\left[H_{n-(1 / 2)}(x)-H_{n+(3 / 2)}\right. \\
& \times(x)]
\end{aligned}
$$

since $d H_{v}(x) / d x=\frac{1}{2}\left[H_{v-1}(x)-H_{v+1}(x)\right]$ (p. 586 in Ref. [31]).
[1] M. D. Levenson and S. S. Kano, Introduction of Nonlinear Spectroscopy (Academic, San Diego, CA, 1988); G. L. Eesley, Coherent Raman Spectroscopy (Pergamon, New York, 1981).

[2] A. H. Zewail, Science 242, 1645 (1988).

[3] M. Schmitt, G. Knopp, A. Materny, and W. Kiefer, Chem. Phys. Lett. 270, 9 (1997).

[4] S. E. Harris, G. Y. Yin, M. Jain, and A. J. Merriam, Philos. Trans. R. Soc. London, Ser. A 355, 2291 (1997); H. Wang, D. Goorskey, and M. Xiao, Phys. Rev. Lett. 87, 073601 (2001); A. B. Matsko, O. Kocharovskaya, Y. Rostovtsev, G. R. Welch, A. S. Zibrov, and M. O. Scully, Adv. At., Mol., Opt. Phys. 46, 191 (2001).

[5] C. Liu, Z. Dutton, C. H. Behroozi, and L. V. Hau, Nature (London) 409, 490 (2001); D. F. Phillips, A. Fleischhauer, A. Mair, R. L. Walsworth, and M. D. Lukin, Phys. Rev. Lett. 86, 783 (2001); A. S. Zibrov, A. B. Matsko, O. Kocharovskaya, Y. V. Rostovtsev, G. R. Welch, and M. O. Scully, ibid. 88, 103601 (2002); A. V. Turukhin, V. S. Sudarshanam, M. S. Shahriar, J. A. Musser, B. S. Ham, and P. R. Hemmer, ibid. 88, 023602 (2002).

[6] S. E. Harris and A. V. Sokolov, Phys. Rev. Lett. 81, 2894 (1998)

[7] M. Jain, H. Xia, G. Y. Yin, A. J. Merriam, and S. E. Harris, Phys. Rev. Lett. 77, 4326 (1996); A. J. Merriam, S. J. Sharpe, M. Shverdin, D. Manuszak, G. Y. Yin, and S. E. Harris, ibid. 84, 5308 (2000); K. Hakuta, L. Marmet, and B. P. Stoicheff, Phys. Rev. A 45, 5152 (1992).

[8] M. O. Scully, G. W. Kattawar, R. P. Lucht, T. Opatrný, H. Pilloff, A. Rebane, A. V. Sokolov, and M. S. Zubairy, Proc. Natl. Acad. Sci. U.S.A. 99, 10994 (2002).

[9] V. A. Sautenkov, C. Y. Ye, Y. V. Rostovtsev, G. R. Welch, and M. O. Scully, Phys. Rev. A 70, 033406 (2004).

[10] V. Kocharovsky, S. Cameron, K. Lehman, R. Lucht, R. Miles, Y. Rostovtsev, W. Warren, G. R. Welch and M. O. Scully, Proc. Natl. Acad. Sci. U.S.A. 102, 7806 (2005).

[11] R. Boyd, Nonlinear Optics (Academic, San Diego, CA, 2003). [12] J. X. Cheng, A. Volkmer, and X. S. Xie, J. Opt. Soc. Am. B
19, 1363 (2002).

[13] P. S. Tuminello, E. T. Arakawa, B. N. Khare, J. M. Wrobel, M. R. Querry, and M. E. Milham, Appl. Opt. 36, 2818 (1997).

[14] J. Kasparian, B. Krämer, J. P. Dewitz, S. Vajda, P. Rairoux, B. Vezin, V. Boutou, T. Leisner, W. Hübner, J. P. Wolf, L. Wöste, and K. H. Bennemann, Phys. Rev. Lett. 78, 2952 (1997).

[15] A. M. Agal'tsov, A. N. Bordeniouk, and V. S. Gorelik, J. Russ. Laser Res. 23, 31 (2002).

[16] A. P. Esposito, C. E. Talley, T. Huser, C. W. Hollars, C. M. Schaldach, and S. M. Lane, Appl. Spectrosc. 57, 868 (2003).

[17] R. Nudelman, B. V. Bronk, and S. Efrima, Appl. Spectrosc. 54, 445 (2000).

[18] N. Wang and H. Rabitz, Phys. Rev. A 52, R17 (1995).

[19] G. Beadie, Z. E. Sariyanni, Y. V. Rostovtsev, T. Opatrný, J. F. Reintjes, and M. O. Scully, Opt. Commun. 244, 423 (2005); Z. E. Sariyanni, and Y. Rostovtsev, J. Mod. Opt. 51, 2637 (2004).

[20] F. M. Kamga and M. G. Sceats, Opt. Lett. 5, 126 (1980); A. Laubereau and W. Kaiser, Rev. Mod. Phys. 50, 607 (1978); W. Zinth, A. Laubereau, and W. Kaiser, Opt. Commun. 26, 457 (1978).

[21] J. D. Jackson, Classical Electrodynamics (Wiley, New York, 1975).

[22] S. E. Harris, Phys. Today 50(9) 36 (1997).

[23] C. F. Bohren and D. R. Huffman, Absorption and Scattering of Light by Small Particles (Wiley, New York, 1983).

[24] E. Hecht and A. Zajac, Optics (Addison-Wesley, New York, 1974).

[25] The linear dependence of the CARS signal on the $\mathcal{R}$ (the Fresnel reflectance) is the manifestation of large quantum coherence. This is because in this case $\widetilde{\rho}_{\mathrm{ac}}^{(3)} \propto \bar{\rho}_{b c} E_{3}$, as seen from Eq. (D4). Here, $\bar{\rho}_{b c}$ does not simply scale as $E_{1} E_{2}^{*}$ (as in the perturbative regime) due to the field dependent denominator $Y(\omega)$ [see Eq. (D5)]. In the perturbative regime, the signal would have scaled as $\mathcal{R}^{3}$.

[26] V. Hizhnyakov and I. Tehver, J. Raman Spectrosc. 28, 403 (1997). 
[27] V. A. Babenko, L. G. Astafyeva, and V. N. Kuzmin, Electromagnetic Scattering in Disperse Media: Inhomogeneous and Anisotropic Particles (Springer, New York, 2003).

[28] M. Rodriguez, R. Bourayou, G. Méjean, J. Kasparian, J. Yu, E. Salmon, A. Scholz, B. Stecklum, J. Eislöffel, U. Laux, A. P. Hatzes, R. Sauerbrey, L. Wöste, and J.-P. Wolf, Phys. Rev. E 69, 036607 (2004).

[29] J. Garduno-Mejia, A. H. Greenaway, and D. T. Reid, Opt. Express 11, 2030 (2003).
[30] For a pulse duration of $\tau_{3}=100 \mathrm{fs}$, energy $10 \mathrm{~mJ}$ and initial beam diameter of $2 \mathrm{~mm}$, the power is $P=0.1 \mathrm{TW}$ and intensity is $3 \times 10^{12} \mathrm{~W} / \mathrm{cm}^{2}$. This is sufficiently high to induce the Kerr effect in long distance propagation. At some distances, the beam diffraction is overcome by self-focusing. Thus, we maintain a small beam diameter of $2 \mathrm{~mm}$ at the spore position.

[31] G. B. Arfken, Mathematical Methods for Physicists, 3rd ed. (Academic, Orlando, 1985). 